$\therefore \quad-\quad+2$

NASA Technical Memorandum 105573
$1 N-78$

79214

$P .30$

\title{
Computational Techniques in Tribology and Material Science at the Atomic Level.
}

J. Ferrante

Lewis Research Center

Cleveland, Ohio

and

G.H. Bozzolo

Analex Corporation

Brook Park, Ohio

Prepared for the

NATO Research Institute Workshop on Fundamentals of Friction sponsored by the North Atlantic Treaty Organization

Brauntage, Germany, August 5-19, 1991 


\title{
COMPUTATIONAL TECHNIQUES IN TRIBOLOGY AND MATERIAL
}

\section{SCIENCE AT THE ATOMIC LEVEL}

\author{
J. Ferrante \\ National Aeronautics and Space Administration \\ Lewis Research Center \\ Cleveland, Ohio 44135 \\ and \\ G. Bozzolo \\ Analex Corporation \\ 3001 Aerospace Parkway \\ Brook Park, Ohio 44142
}

\section{SUMMARY}

Computations in tribology and material science at the atomic level present considerable difficulties. In this paper computational techniques ranging from first-principles to semi-empirical and their limitations are discussed. Example calculations of metallic surface energies using semi-empirical techniques are presented. Finally, application of the methods to calculation of adhesion and friction are presented.

\section{INTRODUCTION}

Computational material science or the theory of materials has recently come of age. Calculation of properties of real materials at the atomic level such as grain boundary or dislocation energies or the dynamics thereof, which in the recent past have seemed intractable, now have some hope for realistic modeling. An even more startling assertion is that modeling of tribological phenomena is also now feasiable. Tribology is a particularly difficult field for theoretical studies at the atomic level because of the different possible materials in contact under high loads often with high degrees of disorder, but as Landman's et al. have shown (paper in this proceedings) a great deal of progress has been made in approaching problems of practical interest. This paper is concerned with developments which have enabled this progress. An effort will be made to define and explain the various approaches for calculating the configurational energy and to give an understanding of such approaches. Finally, we will concentrate on two methods for determining energetics, the embedded atom method (EAM) and equivalent crystal theory (ECT). We concentrate on EAM because it is the method of choice at present and has successfully been used to treat many problems concerning defect energetics. We include ECT, because it is a new method which has the capability of treating a wider class of materials with good quantitative agreement for defect energetics in situations where there is a large deviation from equilibrium. An example of how one might calculate surface energy, adhesive energy, and energy as a function of position for sliding one metal over another will be given with the intent of encouraging the unfamiliar reader to apply these relatively simple techniques to problems of interest.

\section{BACKGROUND}

First, we address the issue of what has changed in atomistic modeling in order to enable examination of problems of interest in tribology. First principles (ab-initio) approaches have dealt with perfect single crystals in which lattice periodicity (ref. 1) reduces the calculation to a single unit cell. The results 
of these calculations were impressively successful, in that accurate agreement with experiment was obtained in predicting the band structure, transport and magnetic properties of solids. Properties of interest to the material scientist, however, are generally related to "defects" in atomic structure which involve a partial loss of periodicity and thus require calculations over a large number of atoms, for example, dislocations, grain boundaries, and interfaces between different materials. The breakdown of periodicity and the involvement of many atoms clearly complicates such problems, for example, we show a twist boundary for an fcc (100) interface (fig. 1). One can see that the geometry is quite complex and that there are many nonequivalent atoms which must be considered in the calculation. Until recently, the lack of adequate affordable computing power combined with lack of sufficiently efficient methods for performing quantum mechanical (ab-initio) calculations made such complex problems difficult. For example, it only recently (refs. 2 and 3 ) became possible to predict which simple structure, fcc, bcc, or hcp for an elemental metal had the lowest energy. These limitations lead to the use of alternative approaches such as pair-potentials (two-body forces) to calculate properties of defects. An additional problem in any approach is that the energies of interest are obtained from the difference in total energy between an ion in the solid and in a reference structure. Each of these quantities is a large number whereas the difference is a small number thus causing a significance problem in obtaining structural energies. We show a simple example (fig. 2) using the jellium model where the charge density of the positive ions in a metal is smeared out uniformly over the solid. The energy difference in creating two surfaces arises from changes in the surface region. The bulk remains the same, thus, integrating over volume with and without the surfaces and subtracting gives a difference in energy small compared to the integral over the entire volume. This situation applies regardless of the approach used to calculate defect energies.

For the next issue, we again refer to figure 1. The rigid twist boundary defect shown is not the minimum energy configuration, consequently a complex search must be performed to find the minimum energy configuration. Finally, if temperature and dynamic effects are to be included the situation becomes even more complex. Having outlined the problem we now examine some approaches.

\section{APPROACHES FOR DESCRIBING THE INTERACTIONS}

In this section we will give a general discussion of approaches for calculating defect energies. The order will be inverted in that we will start with the most general, proceed to the least general, and build to better approximations from the least general. We address four approaches, first-principles calculations, pair-potentials, many-body potentials, and semi-empirical methods. The purpose of these discussions will be to give a qualitative understanding of the methods and to provide a starting point for a literature search should a more comprehensive understanding be desired.

\section{First-Principles Calculations}

This is the most fundamental approach for calculating material properties. In principle, the only inputs are fundamental constants and the atomic number of the atoms of interest and it is not materials limited. Recently, techniques have been developed to treat some of the problems discussed, i.e., loss of periodicity, energy minimization, and dynamic effects. The loss of periodicity is handled by a trick called "super cells" (ref. 4). A quasi-periodicity is produced in which a large cell which mimics the defect is repeated throughout the solid. The hope is that if the cell can be made sufficiently large, the energetics will converge to that of the real defect. Of course in highly disordered systems such as one might find in tribology this approach will not work as well. Carr and Parrinello (ref. 5) have developed a new technique which allows minimization of the total energy as a function of the electronic degrees of freedom and 
also allows the study of dynamic and temperature-dependent effects. However, first-principles calculations remain cpu time intensive.

\section{First-Principles (Ab-initio) Methods}

We now proceed to give a more detailed description of first-principles calculations. These normally involve solving the Kohn-Sham equations within the local density approximation (LDA) (ref. 6). These are a set of equations using the mean-field approximation which are written as a set of one-electron equations where the electron is moving in the mean field of all of the other particles. The total energy is expanded in terms of the electron density, since it can be shown that the total energy is a functional of the electron density. In the LDA only terms that depend on the density at a given position are retained and not terms in the gradient or higher order derivatives. These equations can be written as

$$
\left((-1 / 2) \nabla^{2}+V(\mathbf{r})\right) \Psi_{\mathbf{k}}(\mathbf{r})=\varepsilon_{\mathbf{k}} \Psi_{\mathbf{k}}(\mathbf{r})
$$

where $\Psi_{\mathbf{k}}$ and $\varepsilon_{\mathbf{k}}$ are the one-electron wave functions and energies and $\mathbf{k}$ is the wave number and

$$
\mathrm{V}(\mathbf{r})=\Phi(\mathbf{r})+\mathrm{V}_{\mathrm{xc}}[\rho(\mathbf{r})]
$$

where $\Phi(\mathbf{r})$ is the electrostatic energy $V_{x c}[\rho(r)]$ is the exchange and correlation energy, and $\rho(\mathbf{r})$ is the electron density. Square brackets indicate a functional of the electron density. The wave functions are often expressed in terms of some appropriate basis set (ref. 1). The electrostatic energy is given by

$$
\Phi(\mathbf{r})=\int d \mathbf{r}^{\prime} \rho(\mathbf{r}) / \mathbf{r}-\mathbf{r}^{\prime}\left|\sum_{\mathbf{j}} \mathbf{Z}_{\mathbf{j}} / \mathbf{r}-\mathbf{R}_{\mathbf{j}}\right|
$$

The first term is the electron-electron interaction and the second is the electron-ion interaction, $\mathbf{r}$ is the electron coordinate, $\mathbf{R}$ is the ion coordinate, and $\mathrm{Z}$ is the atomic number. The electron density is given by

$$
\rho(\mathbf{r})=\sum_{\text {occ. }}\left|\psi_{\mathbf{k}}(\mathbf{r})\right|^{2}
$$

where occ. refers to the sum over occupied states. With the geometry and an appropriate approximation for the wave functions, these equations are solved self-consistently, that is, one iterates until the output and input density or potential agree to within some prescribed limit. The total energy is then obtained within the LDA as

$$
\mathrm{E}[\rho]=\mathrm{E}_{\mathrm{KE}}[\rho]+\mathrm{E}_{\mathrm{ES}}[\rho]+\mathrm{E}_{\mathrm{XC}}[\rho]+\mathrm{E}_{\mathrm{ion}-\mathrm{ion}}
$$

where $\mathrm{KE}$ refers to the kinetic energy, ES to the electrostatic energy, $\mathrm{XC}$ to the exchange and correlation energy, ion-ion refers to the ion-ion interaction energy, and the brackets indicate that the total energy is a functional of the electron number density (atomic units are used throughout). Although the first-principles methods are based on the firmest theoretical foundations, there are still many approxi-mations used to get a solution, such as the mean-field approximations and the LDA. Of the methods to be discussed, only ab-initio methods can be used to calculate the electronic structure of the solids and are material independent. 


\section{Pair-Potentials}

In this section we will start with a more general discussion following Carlsson and Ashcroft (ref. 7), which will lead naturally to the following sections. What is the functional form of the cohesive energy $E_{\text {coh }}\left(R_{1}, \ldots, R_{N}\right)$ in a system consisting of $N$ atoms with nuclei at sites $\left\{R_{i}\right\}$ ? A simple guess for expressing the cohesive energy then would be a cluster expansion in pair and higher-order interactions

$$
\begin{aligned}
\mathrm{E}_{\text {coh }}=\mathrm{E}_{1}+ & \mathrm{E}_{2}+\mathrm{E}_{3}+\ldots \\
& =\mathrm{E}_{1}+(1 / 2) \sum_{\mathrm{i}, \mathrm{j}}^{\prime} \mathrm{V}_{2}\left(\mathbf{R}_{\mathrm{i}} \mathbf{R}_{\mathrm{j}}\right)+(1 / 6) \sum_{\mathrm{i}, \mathrm{j}, \mathbf{k}}^{\prime} \mathrm{V}_{3}\left(\mathbf{R}_{\mathrm{i}} \mathbf{R}_{\mathrm{j}} \mathbf{R}_{\mathbf{k}}\right)+\ldots
\end{aligned}
$$

where the prime indicates that no two indeces have the same value and $E_{1}$ represents external forces. The pair-potentials refers to keeping only the $V_{2}$ term in the expansion which dominates when the higher order terms are small in magnitude compared to the pair term. In practice, however, the application of pair-potentials have not followed from such an approach, but by simply assuming some form for $\mathrm{V}_{2}$ and then empirically fitting to some physical parameters, for example, a Morse potential

$$
V_{2}=D\left(\exp \left(-2 \alpha\left(r_{i, j}-r_{0}\right)\right)-2 \exp \left(-\alpha\left(r_{i, j}-r_{0}\right)\right)\right)
$$

The unknown parameters could be selected to give the correct cohesive energy, bulk modulus and equilibrium lattice constant, etc., depending on the number of fitting parameters in the expressions. Once these parameters and the defect geometry are established, the energy is calculated by performing a sum over all pairs of atoms. For some solids such as rare gas solids this approach might be sufficient. In metals, where volume-dependent terms are important (ref. 8), it is not. For example, in figure 3 we show the results of a first-principles calculation for a grain boundary using the jellium model (ref. 9) where the grain boundary is represented by a lower jellium density. We can see that the electron gas has rearranged from the uniform gas. This term would be omitted in the pair-potential approach. There are other deficiencies. For example, they require that the elastic constants satisfy $C_{12}=C_{44}$ which seldom occurs in real metals. In addition, they require that the vacancy formation energy equals the cohesive energy which is also not the case. The vacancy formation energy is typically of the order of one-third of the cohesive energy (refs. 7 and 8) for metals. The principal advantage in pair-potentials is simplicity, however they may be useful in predicting trends when stearic effects dominate.

\section{Many-Body Potentials}

For completeness we now give an example of including the $\overline{\mathrm{V}}_{3}$ term in the series given in equation (6). Stillinger and Weber (ref. 10) have proposed a potential energy function for Si which is constructed to account for the strong directional bonding present in such elements, since pair-potentials had failed to predict behavior for large excursions from equilibrium. They propose for the pair term 


$$
V_{2}(r)=\left\{\begin{array}{l}
A\left(r^{-p}-r^{-q}\right) \exp \left((r-a)^{-1}\right), \quad r<a \\
0, r \geq a
\end{array}\right.
$$

and for $\mathrm{V}_{3}$

$$
\mathbf{v}_{3}\left(\mathbf{r}_{\mathbf{i}}, \mathbf{r}_{\mathbf{j}}, \mathbf{r}_{\mathbf{k}}\right)=h\left(\mathbf{r}_{\mathbf{i j}}, \mathbf{r}_{\mathbf{i k}}, \theta_{\mathbf{j i k}}\right)+h\left(\mathbf{r}_{\mathbf{j i}}, \mathbf{r}_{\mathbf{j k}}, \boldsymbol{\theta}_{\mathrm{ijk}}\right)+\mathrm{h}\left(\mathbf{r}_{\mathbf{k i}}, \mathbf{r}_{\mathbf{k j}}, \boldsymbol{\theta}_{\mathrm{ikj}}\right)
$$

where

$$
\mathrm{h}\left(\mathrm{r}_{\mathrm{ij}}, \mathrm{r}_{\mathrm{ik}}, \theta_{\mathrm{jik}}\right)=\lambda \exp \left(\gamma\left(\mathrm{r}_{\mathrm{ij}}-\mathrm{a}\right)^{-1}+\gamma\left(\mathrm{r}_{\mathrm{ik}}-\mathrm{a}\right)^{-1}\right)\left(\cos \theta_{\mathrm{jik}}+1 / 3\right)
$$

where $h$ vanishes identically for $r \geq a$. The "ideal" tetrahedral angle $\theta_{\mathrm{t}}$ is such that $\cos \theta_{\mathrm{t}}=(-1 / 3)$ so that the trigonometric part of the expression discriminates in favor of pairs of bonds emanating from vertex $i$ with the desired geometry. The fitting parameters were selected through a search which insures that the diamond structure is indeed the most stable arrangement of particles, and that the melting point and the liquid structure inferred be in reasonable agreement with experiment. For a further discussion of the inclusion of higher order terms see references 7 and 8.

\section{Semi-Empirical Methods}

This category of approaches tackles the many-body problem in a different fashion. The term semiempirical refers to determining a functional form for the cohesive energy based on some physical model, but including some parameters determined by fitting to experimental properties. Once these constants are determined, the form is used to calculate the energy or dynamic behavior or other properties of interest such as defect energies. This approach has proven to be extremely effective in accomplishing its goals. This section concentrates on three methods, the method of Finis and Sinclair (refs. 8 and 11) (FS), the embedded atom method (refs. 12 to 14) (EAM), and equivalent crystal theory (refs. 15 to 17) (ECT). The next section concentrates on giving examples from EAM and ECT. The reasons for this idiosyncratic choice is that the authors have no experience in using (FS), however have used EAM and are co-developers of ECT. ECT treats a wide class of materials with a high degree of agreement with experiment and first-principles calculations. EAM has been applied to a wide variety of problems with considerable success.

FS. - Finis and Sinclair (FS) express the total energy of an assembly of atoms in two terms

$$
\mathrm{U}_{\text {tot }}=\mathrm{U}_{\mathrm{N}}+\mathrm{U}_{\mathrm{P}}
$$

where $U_{N}$ is the $\mathrm{N}$-body "cohesive" term and $U_{P}$ is a conventional central pair-potential repulsive term. $U_{N}$ is selected to have the form 


$$
\mathrm{U}_{\mathrm{N}}=-\mathrm{A} \sum_{\mathrm{i}} \mathrm{f}\left(\rho_{\mathrm{i}}\right)
$$

and

$$
U_{P}=(1 / 2) \sum_{i, j} v\left(R_{i j}\right)
$$

where

$$
\begin{gathered}
\rho_{\mathrm{i}}=\sum_{\mathrm{j}} \phi\left(\mathbf{R}_{\mathrm{ij}}\right) \\
\mathbf{R}_{\mathrm{ij}}=\left|\mathbf{R}_{\mathrm{ij}}\right|=\left|\mathbf{R}_{\mathrm{i}}-\mathbf{R}_{\mathrm{j}}\right|
\end{gathered}
$$

The $\phi\left(R_{i j}\right)$ are the electron charge density at site $i$ from an ion atom located at site $j$ and $V\left(R_{i j}\right)$ is a pair repulsion between atoms at site $i$ and $j$. Therefore, equation (11) is interpreted as the sum of $a$ volume dependent term, $U_{N}$, and a pair wise summation $U_{P}$. The term $f(\rho)$ is chosen to be $\sqrt{ } \rho$ in order to mimic the result of tight-binding theory (ref. 1). The unknown constants are obtained from fitting to the cohesive energy, elastic constants, and the bulk modulus. The reader is referred to reference 8 for implementation of the model. Application of the model is similar to methods to be discussed next.

Universality of binding energy relations. - We digress for the moment to discuss an issue which will be important in the discussions of EAM and ECT. Rose, Smith, and Ferrante (refs. 18 and 19) (RSF) discovered that there were similarities between certain binding energy relations, i.e., binding energy versus distance. They found that the functional form of the scaled binding energy curves describing cohesion, adhesion, chemisorption, and diatomics for the case of no charge transfer was the same to a high degree of accuracy (fig. 4). Stated more formally, we write the energy versus separation as

$$
E(R)=\Delta E E^{*}\left(a^{*}\right)
$$

where

$$
\mathrm{a}^{*}=\left(\mathrm{R}-\mathrm{R}_{\mathrm{e}}\right) / \ell
$$

$\Delta \mathrm{E}$ is the binding energy, $\mathrm{a}^{*}$ is a scaled length, $\mathrm{R}$ is the distance between particles, $\mathrm{R}_{\mathrm{e}}$ is the equilibrium distance and $\ell$ is a scaling length. RSF found that a simple functional form was an accurate representation of the function $\mathrm{E}^{*}\left(\mathrm{a}^{*}\right)$ first proposed by Rydberg for diatomics

$$
E^{*}\left(a^{*}\right)=-\left(1+a^{*}\right) \exp \left(-a^{*}\right)
$$

and the length scaling was chosen as

$$
\ell=\left(\Delta \mathrm{E} /\left(\mathrm{d}^{2} \mathrm{E} / \mathrm{dR}^{2}\right)_{\mathrm{R}_{e}}\right)^{-1 / 2}
$$

This result was dubbed the "universal binding energy relation" (UBER). The application of this result to EAM and ECT will be discussed in the next sections. 
EAM. - The embedded atom method (EAM) can be considered as a breakthrough in atomistic simulations of metals. It has been successfully applied to problems such as phonons, liquid metals, defects, fracture, surface structure, surface segregation, etc. (ref. 20). Although it is very similar in structure to FS, the justification for it is different. It is based on the effective medium theory of Stott and Zaremba (ref. 21) or the quasi-atom approach of Norskov and Lang (ref. 22) in which the energy to embed an atom in jellium is calculated. The total energy of the system is written as

$$
\mathrm{E}_{\text {tot }}=\sum_{\mathrm{i}}^{\mathrm{N}} \mathrm{E}_{\mathrm{i}}
$$

where

$$
E_{i}=F_{i}\left(\rho_{h, i}\right)+(1 / 2) \sum_{j \neq i}^{N} \Phi\left(R_{i j}\right)
$$

and $\rho_{h, j}$ is the total electron density at atomic site $i$ due to all the other atoms in the system, $F_{i}$ is the embedding energy for placing an atom at site $i$ and $\Phi\left(R_{i j}\right)$ is a short-range pair-interaction representing the core-core repulsion of atoms $i$ and $j$ separated by $R_{i j}$. N is the total number of atoms in the system. The host electron density at site $i$ is approximated by the linear superposition of all of the other atomic densities

$$
\rho_{h, i}=\sum_{j=i}^{N} \rho_{j}^{a}\left(R_{i j}\right)
$$

where $\rho_{j}^{a}\left(R_{i j}\right)$ is the atomic density of atom $j$ at the distance $R_{i j}$ from its nucleus and

$$
\rho_{\mathrm{j}}^{\mathrm{a}}(\mathrm{R})=\mathrm{n}_{\mathrm{g}} \rho_{\mathrm{g}}(\mathrm{R})+\mathrm{n}_{\mathrm{d}} \rho_{\mathrm{d}}(\mathrm{R})
$$

where $n_{s}+n_{d}$, the total number of $s$ and $d$ electrons, is constrained to be 10 for $\mathrm{Ni}, \mathrm{Pd}$, and $\mathrm{Pt}$; and it is 11 for $\mathrm{Cu}, \mathrm{Pd}$, and $\mathrm{Au}$. The core-core repulsion term takes the form

$$
\Phi\left(R_{i j}\right)=Z_{j}\left(R_{i j}\right) Z_{j}\left(R_{i j}\right) / R_{i j}
$$

where Foiles, Baskes, and Daw have parameterized a simple function for the effective charge $Z(R)$ as

$$
\mathrm{Z}(\mathrm{R})=\mathrm{Z}_{0}\left(1+\beta \mathrm{R}^{\nu}\right) \exp (-\alpha \mathrm{R})
$$

in order to guarantee the short range for the pair interaction, and $\mathrm{Z}_{0}$ is the nuclear charge. Originally, Foiles, Daw, and Baskes (FDB) used a spline fit to define the embedding energy $F(\rho)$, but later found that defect energetics could be better approximated by requiring that for the case of the cohesive energy with an isotropic expansion or contraction of the solid that $F(\rho)$ be required to agree with the UBER (eq. (18)) giving 


$$
F(R)=E_{c o h}(1+\nu x) \exp (-\nu x)-\phi
$$

where $\Phi$ is the second term in equation (21), $E_{\text {coh }}$ is the cohesive energy, $x=\left(r / r_{0}-1\right)$ and $\nu=3\left(\mathrm{~V}_{0} \mathrm{~B}_{0} / \mathrm{E}_{\text {coh }}\right)^{1 / 2} . \mathrm{V}_{0}, \mathrm{~B}_{0}$, and $\mathrm{r}_{0}$ are the equilibrium volume, bulk modulus, and Wigner-Seitz radius, respectively. The unknown constants are again obtained by fitting to such properties as the vacancy formation energy, elastic constants, and diatomic parameters. It is unnecessary to perform the fitting for many materials since they are provided by FDB in their papers. Another point to emphasize is that once the embedding energy is specified for a given metal the same function is then used for any defect. Alloys have been treated in EAM (ref. 23) by keeping the same embedding function for a given metal and constructing the density by the overlap of atomic densities for all species present. For example, in a Ni-Cu alloy, the geometry would be specified, then taking a $\mathrm{Ni}$ site, the overlap contribution to the electronic charge density from both species at the $\mathrm{Ni}$ site is calculated. Finally, that density is used in the $\mathrm{Ni}$ embedding energy function to determine the contribution for the $\mathrm{Ni}$ atom at that site. $\mathrm{A}$ geometric mean of the two pair repulsion terms is used for the interaction between different atoms, i.e.,

$$
\Phi_{A B}(R)=\left[\Phi_{A A}(R) \Phi_{B B}(R)\right]^{1 / 2}
$$

Johnson (ref. 24) has proposed an alternate form for the pair-interaction which gives the same invariance with respect to electron density transformations as monatomic models.

Equivalent crystal theory (ECT). - Equivalent crystal theory is a new technique developed by Smith, Banerjea, and co-workers (ref. 25) which also uses the UBER in order to implement the method. The technique gives quantitatively accurate results for the surface energy and surface relaxation of metals and covalently bonded solids. The application of EAM to bcc metals (ref. 26) and to covalently bonded solids is limited (ref. 27) and thus ECT has fewer material limitations than EAM. The idea is based on the fact that the UBER gives the ground state energy relation for a given solid. Consider first a single crystal of an elemental solid. Next introduce a defect or an array of lattice defects into the crystal. The total energy $\mathrm{E}$ (defect) of the crystal containing the defects is equal to energy of a single crystal $\mathrm{E}$ (crystal) plus a perturbation series, where the perturbing potential is the difference between the array of ion core potentials of the crystal containing defects and those of the single crystal:

$$
\mathrm{E}(\text { defect })=\mathrm{E}(\text { crystal })+\text { Perturbation series }
$$

$\mathrm{E}\left(\right.$ crystal) is given by equation (16) where $\mathrm{a}^{*}=\left(\mathrm{r}_{\mathrm{WS}}-\mathrm{r}_{\mathrm{WSE}}\right) / 1, \mathrm{r}_{\mathrm{WSE}}$ is the equilibrium Wigner-Seitz radius $\left[3 /\left(4 \pi \mathrm{r}_{\mathrm{WSE}}\right) \equiv\right.$ bulk atom density $], \mathrm{l}=\left[\Delta \mathrm{E} /\left(12 \pi \mathrm{Br}_{\mathrm{WSE}}\right)\right]^{1 / 2}$ and $\mathrm{B}$ is the bulk modulus of the ground state single crystal. At this point we present the idea of the "equivalent crystal." The equivalent crystal is a conceptually ideal crystal of the material which is either expanded or con-tracted from the ground state crystal and thus its energy is given by the UBER at some different scaled distance, $a_{0}^{*}$. The procedure is to find the value of $a_{0}^{*}$ for which the perturbation series $=0$. If this can be accomplished then

$$
\mathrm{E}(\text { defect })=\Delta \mathrm{E} \mathrm{E}^{*}\left(\mathrm{a}_{0}^{*}\right)
$$

which can be evaluated simply by using the UBER. In order to implement ECT, we must have a method to represent the perturbation series. As before, the purpose of this presentation is to show how to use ECT, hence no formal justification will be given and the reader is referred to references 25 and 17 for this aspect. The perturbation series has matrix elements which are integrals over products of the density and 
the difference in potential between the perturbed and unperturbed state. These integrals are approximated by

$$
g \propto R^{\mathbf{P}} \exp (-\alpha R)
$$

for nearest neighbors and

$$
g \propto R^{p} \exp (-\alpha R) \exp (-R / \lambda)
$$

for next nearest neighbors and beyond, where $p=2 n-2, n=$ principal quantum number and $\lambda=$ electronic screening length. It is assumed that the form of the electron density is that of the highest partially occupied $s$ orbital. Screening is introduced for next nearest neighbors by addition of the term

$\exp (-\mathrm{R} / \lambda)$. The calculation proceeds for solving the perturbation equation on a site by site basis, so that the energy change is written as the sum over the changes at each nonequivalent site given by

$$
\delta \mathrm{E}=\sum_{\mathrm{l}=1}^{\mathrm{s}}\left[\mathrm{E}_{\mathrm{l}}(\text { defect })-\mathrm{E}_{\mathrm{l}}(\text { crystal })\right]
$$

where the $E_{l}$ 's are the energies associated with site 1 for the defect crystal and the ground state crystal, respectively. In many defects where a high degree of symmetry is maintained such as a surface, the value of $s$ in equation (22) can be quite small. We now present an older version of ECT which is sufficient to calculate surface energy and surface relaxation for our example calculation. The more general method will be outlined after this discussion. We now introduce the actual working equations for application of the perturbation equation (32) for $\delta \mathrm{E}=0$ for a given site 1 , to next nearest neighbors

$$
\begin{aligned}
b_{1} R_{1}^{p}(1) e^{-\alpha R_{1}(1)}+b_{2} R_{2}^{p} e^{-\alpha R_{2}(1)} e^{-R_{2}(1) / \lambda} & =\sum_{\text {defect } n} R_{i}^{\prime p}(1) e^{-\alpha R_{i}^{\prime}(1)} \\
& +\sum_{\text {defect nn }} R_{i}^{\prime p} e^{-\alpha R_{i}^{\prime}(1)} e^{\cdot R_{i}^{\prime}(1) / \lambda}
\end{aligned}
$$

where $b_{1}$ and $b_{2}$ are the number of nearest (next) neighbors, respectively; and $R_{1}$ and $R_{2}$ are the nearest(next) neighbor distances, respectively. We solve this equation for $R_{1}$, the equivalent crystal nearest neighbor distance, which has a simple geometric relationship to the Wigner-Seitz radius and all of the neighbor distances since we are dealing with a perfect crystal. To restate, an atom in a defect in the material experiences an environment as if it were in a perfect crystal, which is expanded or contracted from its equilibrium state to a new nearest neighbor distance given by $R_{1}$. The expression for the surface energy, for which we will give an example later, is given by

$$
\sigma=(\Delta \mathrm{E} / \mathrm{A}) \sum_{\mathrm{l}=1}^{\mathrm{s}}\left[\mathrm{F}^{*}\left(\mathrm{a}_{01}^{*}\right)+\sum_{\mathrm{m}=1}^{\mathrm{M}} \Theta_{\mathrm{ml}} \mathrm{F}^{*}\left(\mathrm{a}_{\mathrm{ml}}^{*}\right) / \mathrm{L}_{\mathrm{ml}}\right]
$$

where 


$$
\mathrm{F}^{*}\left(\mathrm{a}^{*}\right)=1+\mathrm{E}^{*}\left(\mathrm{a}^{*}\right)=1-\left(1+\mathrm{a}^{*}\right) \mathrm{e}^{-\mathrm{a}^{*}}
$$

where $\mathbf{A}$ is the surface area, $s=$ number of layers with energy different from the bulk $(\approx 6)$, $M_{*}=$ number of nearest neighbors for layer $1, \Theta_{\mathrm{ml}}=1$ if $\mathrm{a}_{\mathrm{ml}} \leq 0$ and $\Theta_{\mathrm{ml}}=0$ otherwise, $a_{m 1}^{*}=R_{1}(1) / c_{1}-r_{W S E}\left(c_{1}\right.$ is the ratio between the nearest neighbor distance and the Wigner-Seitz radius), $a_{m l}^{*}=R_{m l} / c_{1}-r_{W S E}, R_{m l}$ is the distance between atoms $m$ and $l$, and $L_{m l}$ is the number of nearest neighbors of atom $\mathrm{m}$ or $\mathrm{l}$, whichever is smaller. The first term in equation (34) gives the deviation from the minimum in the binding energy curve and thus the increase in energy from being in the defect. The second term is included to deal with the fact that when relaxation is included bonds are distorted inhomogeneously, as opposed to the first term which depends on the average nearest neighbor distance. The second term is zero when bonds are not compressed. For the rigid surface energy (i.e., no configurational relaxation) the second term can be neglected. ECT in this form has essentially only one fitting parameter, $\alpha$, which is obtained from the vacancy formation energy. The other physical parameters such as the cohesive energy, bulk modulus, and equilibrium lattice parameter appear explicitly in the model.

Since the purpose of this presentation is to give an overview of the methods, we will not go into details for the modification of ECT so that a wider class of materials and situations can be treated. We will only indicate the general procedures leaving the details to reading reference 25 . The defect formation energy $\varepsilon_{i}$ is now written in terms of four components:

$$
\varepsilon_{\mathrm{i}}=\Delta \mathrm{E}\left\{\mathrm{F}^{*}\left[\mathrm{a}_{1}^{*}(\mathrm{i})\right]+\mathrm{F}^{*}\left[\mathrm{a}_{2}^{*}(\mathrm{i})\right]+\sum_{\mathrm{j}, \mathrm{k}} \mathrm{F}^{*}\left[\mathrm{a}_{3}^{*}(\mathrm{i}, \mathrm{j}, \mathrm{k})\right]+\sum_{\mathrm{p}, \mathrm{q}} \mathrm{F}^{*}\left[\mathrm{a}_{4}^{*}(\mathrm{i}, \mathrm{p}, \mathrm{q})\right]\right\}
$$

This expression is now in the spirit of the many-body expansion presented in equation (6). Note that for the case of surface relaxation that equation (34), which is a simpler version of equation (36), suffices. In this approach each term is treated as though it is an independent perturbation. The first term is that presented in the original formulation of ECT, the second, the bond-compression term, although formulated differently, has the same purpose as already presented. The third term is included to handle the situation where bond angle changes are important as in semi-conductors. The fourth term is included in order to give the correct values for the shear elastic constants. In this formulation there are four fitting parameters compared to one, $\alpha$. These four parameters are obtained from the vacancy formation energy and the elastic constants. The other input parameters remain as before. The procedure is more complicated but not difficult, because each perturbation is treated as independent. There are now four equations similar to equation (33) but no more difficult than it to solve for the a 's. The results for EAM and ECT will be shown in a later section. Finally, alloys (ref. 28) are treated by an extension of the basic ECT ideas in which the $\alpha$ 's reflect whether the neighbors are of type $A$ or $B$.

\section{EXAMPLES OF SURFACE ENERGY CALCULATIONS}

We now give an example of a surface energy calculation for a rigid fcc (100) metal surface using EAM and ECT to demonstrate the relative simplicity of such calculations. Each atom in each plane is equivalent to all other atoms in the plane, therefore it is only necessary to solve the EAM or ECT equations for a single atom in each plane and the only remaining question is how many planes are needed for convergence. In EAM, the first step is to pick an atom in the plane, say atom $A$, and then evaluate the electron density at $A$ from all of the other atoms in the crystal (eqs. (22) and (23)). The density is evaluated 
from analytic expressions (ref. 12). Once the density is known, one determines the embedding function, $\mathrm{F}(\rho)$ (eq. (20)), for the given metal at that density. Then one need only evaluate the pair term in equation (20) using the parameters given in reference 12 and then evaluate the contribution to the energy for this atom from equation (20). The calculation is finished for the top plane. The same calculation is repeated for a single atom in the second plane. This procedure is continued in each plane until there is no change in the energy from the bulk value. For EAM one would subtract the bulk energy for each atom, sum and divide by the area of a primitive cell in order to calculate the surface energy, i.e.,

$$
\sigma=\sum_{i}^{s}\left(E_{i}^{\text {plane }}-E_{\text {bulk }}\right) / A
$$

We now outline the procedure for doing the same calculation with ECT. Again we start with the same picture, an atom in the surface plane. We will only concern ourselves with up to next nearest neighbors in this example. Referring to equation (33), the perfect fcc equivalent crystal has 12 nearestneighbors and 6 next nearest-neighbors, therefore $b_{1}=12$ and $b_{2}=6$. An atom in the free surface has lost four nearest-neighbors and one next nearest neighbor, thus we can rewrite equation (33) for the surface atom as

$$
12 R_{1}^{p}(1) e^{-\alpha R_{1}(1)}+6 R_{2}^{p}(1) e^{-\alpha R_{2}(1)} e^{-R_{2}(1) / \lambda}=8 R_{1}^{\prime} p(1) e^{-\alpha R_{1}^{\prime}(1)}+5 R_{2}^{\prime} p(1) e^{-\alpha R_{2}^{\prime}} e^{-R_{2}^{\prime}(1) / \lambda}
$$

An atom in the second plane keeps all of its nearest-neighbors and loses one next nearest-neighbor, therefore we have

$12 R_{1}^{P}(2) e^{-\alpha R_{1}(2)}+6 R_{2}^{P}(2) e^{-\alpha R_{2}(2)} e^{-R_{2}(2) / \lambda}=8 R_{1}^{\prime}(2) e^{-\alpha R_{1}^{\prime}(2)}+5 R_{2}^{\prime P}(2) e^{-\alpha R_{2}^{\prime}} e^{-R_{2}^{\prime}(2) / \lambda}$

where now the arguments of $R$ refer to the equivalent atom in each plane and noting that $R_{2}$ is simply a geometrical factor times $R_{1}$ and $R^{\prime}$ is known since we are specifying the geometry of the defect as are $\alpha$ and $\lambda$, which are given in the ECT papers. Thus, we are simply left with the problem of solving each transcendental equation for $R_{1}$ for each nonequivalent atom in the surface region. Once this is done we simply substitute the values into equation (34) and evaluate the energy, where, for the rigid crystal, only the first term contributes. It is conceivable that for high degrees of symmetry and truncating at next-nearest neighbors that the calculation can be done on a programmable hand calculator.

Let's consider how many terms must be kept for the surface energy calculation. Note that the cpu time consuming step in EAM is overlapping the densities, and in ECT it is solving the transcendental equation. Table I shows the contributions to the surface energy for EAM and ECT for a $\mathrm{Ni}(100)$ surface. In either case, three planes are sufficient to evaluate the energy. Table II shows the ECT values for an $\mathrm{Al}(210)$ surface. Since this is a high index plane, lower lying planes can have neighbors in the surface layer and thus more planes must be kept in the calculation.

In table III we show a comparison between ECT, EAM, first-principles and experiment for the surface energies. ECT gives quantitatively accurate agreement with first-principles calculations (typically $<10$ percent disagreement) whereas EAM gives about 40 percent disagreement. In addition, ECT can be simply used for any crystal structure whereas EAM has been primarily applied to fcc metals although it 
has been used for bcc and hcp in some cases. In table IV we show a comparison for surface relaxation and again ECT reproduces the magnitudes and trends quite well. This is a very stringent test, since the energy differences are very small in this case. In table $\mathrm{V}$ we show the results of using the more general form of ECT (eq. (36)) applied to Si.

It is evident that EAM underestimates the surface energy. Our opinion is that these effects are caused by the fact that overlapping atomic densities does not include relaxation of the electron gas. At a free surface there are large deviations from equilibrium using simple overlap of free atom densities, whereas for an internal interface such as a grain boundary simple overlap is a less severe approximation and the energies obtained may be quite good. ECT has in some sense, electronic relaxation built into the procedure of finding the equivalent crystal and thus avoids this difficulty. Since the energy depends variationally on errors in the electron density, errors in the density only cause second order errors in the energy. In this section, it is hoped that we have communicated that the application of EAM and ECT to problems of interest can be relatively simple.

\section{FRICTION AND OTHER STUFF}

Although these proceedings involve friction, we are going to avoid the real issue of how do you model the loss processes and instead present results of another problem which is a bound on the friction process, i.e., sliding one single crystal surface over another and also discuss a recent first-principles calculation of slip.

We start with a general discussion of the adhesion by referring back to figure 2. Here we show a solid which is separated perdendicular to some plane. If we calculate the total energy at each separation, the adhesive binding energy is defined as

$$
\mathrm{E}_{\mathrm{AD}}(\mathrm{a})=(\mathrm{E}(\mathrm{a})-\mathrm{E}(\infty)) / 2(2 \mathrm{~A})
$$

where $\mathbf{a}$ is the separation between surfaces and $\mathbf{A}$ is the cross-sectional area. Figure 5(a) shows what general shape one would expect the curves to have. Taking the derivative of this curve, the tensile stress is the curve shown in figure 5(b). Figure 6 shows the results of a first-principles, jellium calculation performed by Ferrante and Smith (ref. 29), solving equations (1) to (5) for this approximation. We can see that the curves for different metals in contact have large binding energies and have the same features as the same metal contacts. Figure 7 shows that all of these curves scale onto one binding energy relation: the UBER described earlier. We now show adhesion calculations using ECT. The adhesion calculation for ECT or EAM is essentially as simple as the surface energy calculation. The only difference is that the other half space is present and its position is changed. Figure 8(a) shows (refs. 30 and 31) the results of an adhesion calculation for the (111) surface of a number of fcc metals and figures 8 (b) and (c) show the adhesive energy curves for the (110) surface of two bcc metals. The corresponding scaled binding energy curves are presented in figures 9 (a) and (b) along with a fit to the Rydberg function. We can see that the curves scale. Figure 10 gives the force as a function of separation for (110) iron and tungsten. ECT can be formulated completely in terms of force, there-fore these calculations can be done directly. ECT calculations are trivial compared to the jellium or fully three-dimensional first-principles results presented.

An interesting aspect of the adhesion calculation which was ignored in previous calculations relates to instabilities which can occur as surfaces approach on another called "avalanche." Studies of this topic were prompted by a paper by Pethica and Sutton (ref. 32). The authors using ECT (ref. 33) and planar surfaces extended their results. The assumption that adhesion could take place with the half spaces 
essentially rigid with possibly some small relaxation is clearly not correct. We found, as did Pethica and Sutton, that at a certain separation the surfaces will snap together depending on the thickness of the metals in contact. In retrospect this result is not too surprising since the spring constant for the restoring force depends on the length of the solid. Figure 11 shows the energy for moving one surface layer as a function of separation. At a separation of $2.6 \AA$ there is a barrier to moving one layer from each surface whereas at $1.9 \AA$ this barrier is removed. Figure 12 shows the energy as a function of separation for relaxing a number of surface layers where the circles represent the "rigid adhesion" curves from our previous calculations. As the number of planes relaxed (length of the solid) is increased, "avalanche" occurs at larger separations. Figure 13 shows how the separation at which avalanche occurs depends on the number of layers relaxed and the rigid separation. This example demonstrates that these calculational methods enable examination of phenomena which are difficult to observe experimentally.

We now address some ECT results that model friction more closely, that of the slip of one halfspace over another (ref. 34). We have used ECT to calculate the energy and force as a function of tangential position for slip on an fcc (100) surface for a number of metals. This calculation was performed under zero applied load conditions, i.e., as a force tangential to the surface was applied the two halfspaces were allowed to breath at the separation plane in the direction normal to the (100) surface. Once this calculation was finished the results were fitted to a Fourier series for the energy with a modulating term due to the normal displacement given by

$$
\sigma(\mathbf{r})=\sum_{\mathrm{i}=0}^{2} \Delta_{\mathrm{i}}\left(1+\mathrm{z}_{\mathrm{i}}+\beta_{\mathrm{i}} \mathrm{z}_{\mathrm{i}}^{3}\right) \mathrm{e}^{-\mathrm{z}_{\mathrm{i}}} \mathrm{H}_{\mathrm{i}}(\mathrm{x}, \mathrm{y})
$$

where

$$
\begin{gathered}
\mathrm{z}_{\mathrm{i}}=\left(\mathrm{z}-\mathrm{z}_{0}^{\mathrm{i}}\right) / \mathrm{l}_{\mathrm{i}} \\
\mathrm{H}_{0}(\mathrm{x}, \mathrm{y})=1-\mathrm{H}_{1}(\mathrm{x}, \mathrm{y}) \\
\mathrm{H}_{1}(\mathrm{x}, \mathrm{y})=\cos (2 \pi \mathrm{x} / \mathrm{a}) \cos (2 \pi \mathrm{y} / \mathrm{a}) \\
\mathrm{H}_{2}(\mathrm{x}, \mathrm{y})=\cos (4 \pi \mathrm{x} / \mathrm{a})+\cos (4 \pi \mathrm{y} / \mathrm{a})-2 \mathrm{H}_{1}(\mathrm{x}, \mathrm{y})
\end{gathered}
$$

where $a$ is the lattice parameter and $\Delta_{i}, B_{i}$, and $l_{j}$ are fitting constants from the ECT calculations and are given in reference 34. Equation (41), which can be thought of as an extension of the Frenkel model (ref. 1), is an excellent representation of the numerical results, with average deviation of order $10^{-4} \mathrm{eV}$ per surface atom for all metals treated. This is illustrated in figures 13 and 14 where the numerical results of equations (41) to (45) are plotted as solid curves. Figure 13 shows ideal adhesion or cleavage as a func-tion of $\mathrm{z}$ at fixed $(\mathrm{x}, \mathrm{y})$ for the $\mathrm{Ag}(100)$ interface. The bottom curve corresponds to $(\mathrm{x}, \mathrm{y})=$ $(0,0)$, the middle to $(a / 4,0)$ and the top to $(a / 2,0)$.

Equations (41) to (45) and figure 14 apply to arbitrary slip directions where the repeat distance can be many times the lattice constant a. Figure 14 shows zero-load results for two illustrative slip directions, the lower curve shows results for slip along the $\mathrm{x}$-axis and in the upper an angle of arctan $(3 / 4)$ relative to the $\mathrm{x}$-axis. Figure 14 also shows the scaled results for all four metals examined where the energy was divided by the surface energy, $\Delta_{1}$, and the distance by the lattice parameter, a. Thus there appears to be a universal form for ideal slip. This universality leads to useful rules of thumb for 
slip, analogous to the Griffith criterion for cracks. Consequently, we find that the average energy barrier to slip is $E_{m}=0.36 \Delta_{1}$ and the average maximum slip stress is $\sigma_{\mathrm{m}}=1.45 \Delta_{1} / \mathrm{a}$.

Figure 15 provides an overall picture of the stress profile for an Ag interface sliding on the (100) surface at zero load. The size of the arrow head and the length of the vector are both proportional to the magnitude of the slip stress. In table VI we show the numerical results for the height of the energy barrier and the maximum shear stress in the direction of minimum resistance to slip along with a comparison with whisker strengths. Although the theoretical results are larger, they are of the same order of magnitude as the experimental results. Since in the fcc system slip occurs on (111) planes, the results are in reasonable agreement. The ability to map the entire energy surface and then analyze the results for other relationships shows the power of the semi-empirical methods. What we have presented here is not friction, however, since there are no energy dissipation mechanisms in the calculation. In addition, at this stage of the calculation we have ignored relaxation and any dynamic effects that will occur during sliding. However, the minimum stress to initiate sliding is a number of interest and the fact that scaling was found may mean that there are some underlying simplicities in the mechanisms.

We now discuss a first-principles calculation similar to the above of sliding at a Pd-graphite interface by Zhong and Tomanek (ref. 35). They calculate the force to slide a mono-layer of Pd in registry with the graphite surface. Instead of a zero load, they calculate the sliding force as a function of load. Figure 16 shows their results for the position of the potential energy barrier and tangential force as a function of sliding distance in a given direction from hollow to bridge sites. The results are obtained from solving the Kohn-Sham equations (eqs. (1) to (5)) for the situation described. The effects of the load are obtained by calculating the adhesive energy, i.e., the energy as a function of distance in the normal direction. No specific mechanism is presented for energy dissipation and the assumption is made that all of the energy gained in being pushed up the hill is efficiently transferred into creating surface phonons and electron-hole pairs. Zhong and Tomanek then assume that the energy dissipated is given by

$$
\Delta E_{f}=\left\langle f_{f}\right\rangle \Delta x
$$

where $\left\langle f_{f}\right\rangle$ is the average friction force in sliding a distance $\Delta x$. The energy dissipated is estimated from the maximum barrier height in a given direction (fig. 16), and thus $\Delta \mathrm{E}_{\mathrm{f}}=\Delta \mathrm{V}_{\max }$ and thus the average friction coefficient is given by the average friction force divided by the external load as

$$
\mu=\left\langle\mathrm{f}_{\mathrm{f}}\right\rangle / \mathrm{f}_{\text {ext }}=\Delta \mathrm{V}_{\max } /\left(\mathrm{f}_{\mathrm{ext}} \Delta \mathrm{x}\right)
$$

A plot of this result is given in figure 17. The apparent anomaly for low load is a result of the minimum in the potential energy switching from a hollow to a bridge site under load. They then argue that the value obtained $\mu \simeq 0.02$ is in agreement with experiment.

One final topic we should address is ceramic and ionic compounds, since tribology has a wide range of materials involved in sliding contact and ceramic compounds are becoming increasingly important in newer high-temperature engines. At present pair-potentials (refs. 36 and 37) are the forms most often used to model interfaces and other defects. This approach suffers from the same difficulties previously described with the added complexity of having the possibility of having net charges at interfaces. Development of new approaches to treat these problems is definitely a goal for the future.

First-principles calculations are presently being used to treat the problem of ceramic interfaces with their advantage of not being material dependent, but with the limitation that super-cells are used. As an example of such calculations we show a super-cell (fig. 19) structure used to calculate the interfacial 
energy for a diamond C/sphalerate Boron-Nitride interface (ref. 38) using a linear-muffin-tin-orbital-approach (LMTO). The calculated adhesive energies range from 0.89 to $0.95 \mathrm{eV} /$ unit cell area, depending on what structure is assumed for the interface. These calculations did not include any relaxation of the structure. These structural relaxations can be done if the changes are mainly local, which is probably the case. The first-principles calculations give promise for providing information concerning interfacial energies at least for the materials combinations of interest in tribology.

At present there are no semi-empirical approaches for nonmetals. Recently, we have proposed (refs. 39 and 40) an expression for the total energy for situations with charge transfer such as ionic solids with the hope that a method could be developed that would treat ceramic materials. The expression we propose is given by

$$
E(R)=-C\left(1+a^{*}\right) e^{-a^{*}}-\alpha \delta Z^{2} f(R) / R
$$

where the first term allows for the possibility of both covalent bonding and the repulsion under bonding and the second the coulombic charge transfer, where $C$ is the well depth of the Rydberg part, $a^{*}$ is now $\left(R-R_{E}^{\prime}\right) / l$ and $R_{E}^{\prime}$ is the distance at which the minimum occurs for the Rydberg part, $\delta \mathrm{Z}$ is the charge transfer, $\alpha$ is the modeling constant, and $f(R)$ is a function that accounts for the crossing of the energy from two ions to two neutrals at some distance. This result can be contrasted with a Born-Mayer potential (ref. 1) which is a similar expression, but has a repulsive exponential core and thus does not allow for the possibility of covalent bonding. Figure 20 shows a fit of this function to the potential energy curves from first-principles calculations for three ionic molecules, $\mathrm{AlCl}, \mathrm{AlF}$, and $\mathrm{LiF}$ ( $\alpha=1$, for this case). In addition to the fits being quite good the values obtained for the charge transfer, the relative covalent contribution, and the spectroscopic constants obtained from the global fits are quite reasonable. The hope in pursuing these studies is that a semi-empirical method can be developed similar to EAM or ECT in order to treat nonmetal and metal-nonmetal defects.

\section{CONCLUDING REMARKS}

In this presentation we have tried to give a description of the various computational techniques presently being used to approach problems in computational material science. We have also indicated that such calculational techniques now are sufficiently accurate and along with improvements in computer speed enable serious consideration for attacking problems in tribology for certain classes of materials. At present it seems unlikely that the configurational energy minimization and dynamics needs for modeling tribological problems will be amenable to first-principles approaches because of the complexity of the problem and the computer time needed to treat each configuration. A more likely scenario will be the development of semi-empirical approaches which give accurate values for defect energies as compared to static configuration first-principles calculations and are then used to treat configurational energy minimization and dynamic effects.

\section{REFERENCES}

1. Ashcroft, N.W. and Mermin, N.D., Solid State Physics, pub. Holt, Rinehart and Wilson, New York, 1976.

2. Skriver, H.L., Phys. Rev. Lett. 49, 1768 (1982). 
3. Yin, M.T., and Cohen, M.L., Phys. Rev. B 26, (1982).

4. Vicenzo, D.P., Alehand, D.L., Schlutter, M., and Wilkins, J.W., Phys. Rev. Lett. 56, 1925 (1986); See also Payne, M.W., Bristowe, P.D., and Johannapoulis, J.D., Phys. Rev. Lett. 58, 1348 (1987).

5. Carr, R., and Parrinello, M. Phys. Rev. Lett. 55, 2471 (1985).

6. Kohn, W., and Sham, L.J., Phys. Rev. A 140, 1133 (1965).

7. Carlsson, A.E. and Ashcroft, N.W., Phys. Rev. B 27, 2101, (1983).

8. Finnis, M.W. and Sinclair, J., Phil. Mag. A 50, 45 (1984).

9. Smith, J.R. and Ferrante J., Phys. Rev. B 31, 3427 (1988).

10. Stillinger, F. H. and Weber, T.A., Phys Rev. B 31, 5262 (1985).

11. Sutton, A.P., Finnis, M.W., Pettifor, D.G., and Ohta, Y.J. Phys. C 21, 35 (1988).

12. Foiles, S.M., Baskes, M.I., and Daw, M.S., Phys. Rev. B 33, 7983 (1986).

13. Foiles, S. M., Phys. Rev. B 32, 3409 (1985).

14. Daw, M.S., Phys. Rev. B 39, 7441 (1989).

15. Smith, J.R. and Banerjea, A., Phys. Rev. Lett. 59, 2451, (1987).

16. Smith, J.R. and Banerjea, A., Phys. Rev. B 37, 1041 (1988).

17. Smith, J.R., Perry, T.A., and Banerjea, A., in Atomistic Simulations Beyond Pair-Potentials, eds. V. Vitek and D.J. Srolovitz (Plenum Press, N.Y. 1989) p. 279.

18. Rose, J.H., Smith, J.R., and Ferrante, J., Phys. Rev. B 28, 1835 (1983).

19. Rose, J.H., Ferrante, J., and Smith, J.R., Phys. Rev. Lett. 47, 675 (1981).

20. Foiles, S.M., Phys. Rev. B 32, 7685 (1985).

21. Stott, M.J. and Zaremba, E., Phys. Rev. B 22, 1564 (1980).

22. Norskov, J.K. and Lang, N., Phys. Rev. A 26, 2875 (1982).

23. Foiles, S.M. and Daw, M.S., J. Mat. Res. 2,5 (1987).

24. Johnson, R.A., Phys. Rev. B 39, 2554 (1989).

25. Smith, J.R., Perry, T.A., Banerjea, A., Ferrante, J., and Bozzolo, G., Phys Rev. B 44, 6444 (1991).

26. Willaime, F. and Massobrio, C., Phys. Rev. B 43, 11653 (1991). 
27. Baskes, M.I., Phys. Rev. Lett. 59, 2666 (1987).

28. Bozzolo, G. Ferrante, J., and Smith, J.R., Phys. Rev. B45, 493 (1992).

29. Ferrante J. and Smith, J.R., Phys. Rev. B 31, 3427 (1985).

30. Banerjea, A., Ferrante, J., and Smith, J.R., J. Phys: Cond. Mat. 2, 8841 (1990).

31. Banerjea, A., Ferrante, J., and Smith, J.R., Fundamentals of Adhesion, (ed. L.-H Lee, Plenum, New York, 1991) p. 325.

32. Pethica, J.B. and Sutton, A., J. Vac. Sci. Tech. 6, 2490 (1988).

33. Smith, J.R., Bozzolo, G., Banerjea, A., and Ferrante, J., Phys. Rev. Lett. 63, 1269 (1989).

34. Bozzolo, G., Ferrante, J., and Smith, J.R., Scripta Met. et Mat. 25, 1927 (1991).

35. Zhong, W. and Tomanek, D., Phys. Rev. Lett. 64, 3054 (1990).

36. Catlow, C.R.A., Diller, K.M., and Nagett, M.J., J. Phys. C: Solid State 10, 1395 (1977).

37. Duffy, D.M., J. Phys. C.: Sollid State 19, 4393 (1986).

38. Lambrecht, W.R.L. and Segall, B., Phys. Rev. B 40, 7793 (1989).

39. Ferrante, J., Schlosser, H., and Smith, J.R., Phys. Rev. A 43, 3487 (1991).

40. Smith, J.R., Schlosser, H., Leaf, W. Ferrante, J., and Rose, J.H., Phys. Ref. A 39, 925 (1989).

TABLE I. - PLANAR CONTRIBUTION

TO THE SURFACE ENERGY USING

EAM AND ECT FOR Ni(100)

\begin{tabular}{|c|c|c|}
\hline Plane & EAM & ECT \\
\hline 1 & $0.290 \mathrm{eV} /$ atom & $\begin{array}{c}0.654 \mathrm{eV} / \text { atom } \\
1.25 \times 10^{-6}\end{array}$ \\
\hline
\end{tabular}

TABLE II. - PLANAR CONTRIBUTION

TO THE SURFACE ENERGY USING

ECT FOR Al (210)

\begin{tabular}{|c|c|}
\hline Plane & ECT \\
\hline 1 & $1.25 \mathrm{eV} /$ atom \\
2 & 0.404 \\
3 & 0.055 \\
4 & $1.25 \times 10^{-6}$ \\
\hline
\end{tabular}


TABLE III. - RIGID AND RELAXED SURFACE ENERGIES IN $\mathrm{erg} / \mathrm{cm}^{2}$ FROM ECT, FIRST-PRINCIPLES AND EAM (OTHER) CALCULATIONS IN REFERENCE 33

\begin{tabular}{|c|c|c|c|c|c|}
\hline Element & Crystal face & ECT rigid & ECT relaxed & LDA rigid & Other \\
\hline \multirow[t]{3}{*}{$\mathrm{Cu}$} & (111) & 1830 & 1780 & 2100 & 1170 \\
\hline & $(100)$ & 2380 & 2320 & 2300 & 1280 \\
\hline & $(110)$ & 2270 & 2210 & & 1400 \\
\hline \multirow[t]{3}{*}{$\mathbf{A g}$} & (111) & 1270 & 1230 & & 620 \\
\hline & $(100)$ & 1630 & 1600 & 1650 & 705 \\
\hline & $(110)$ & 1540 & 1510 & & 770 \\
\hline \multirow[t]{3}{*}{$\mathrm{Ni}$} & (111) & 2400 & 2320 & & 1450 \\
\hline & $(100)$ & 3120 & 3040 & 3050 & 1580 \\
\hline & $(110)$ & 2980 & 2910 & & 1730 \\
\hline \multirow[t]{3}{*}{$\mathrm{Al}$} & (111) & 920 & 860 & & \\
\hline & $(100)$ & 1290 & 1220 & & \\
\hline & (110) & 1310 & 1230 & 1100 & \\
\hline \multirow[t]{2}{*}{$\mathrm{Fe}$} & (110) & 1820 & & & \\
\hline & $(100)$ & 3490 & & 3100 & 1693 \\
\hline \multirow[t]{2}{*}{ W } & (110) & 3330 & & & \\
\hline & (100) & 5880 & & 5200 & 2926 \\
\hline
\end{tabular}


TABLE IV. - PERCENTAGE CHANGES IN INTERLAYER SPACING DUE TO RELAXATION, REFERENCES FOR

EACH VALUE ARE GIVEN IN REFERENCE 33

\begin{tabular}{|c|c|c|c|c|c|}
\hline Element & $\Delta d_{n, m+1}$ & $\begin{array}{c}\text { ECT, } \\
\%\end{array}$ & $\begin{array}{c}\text { EAM, } \\
\%\end{array}$ & $\begin{array}{c}\text { Experiment, } \\
\%\end{array}$ & Technique \\
\hline $\mathrm{Cu}(110)$ & $\begin{array}{l}\Delta d_{12} \\
\Delta d_{23}\end{array}$ & $\begin{array}{l}-7.7 \\
+3.4\end{array}$ & $\begin{array}{l}-4.9 \\
+0.2\end{array}$ & $\begin{array}{l}-8.5 \pm 0.6 \\
-7.5 \pm 1.5 \\
+2.3 \pm 0.8 \\
+2.5 \pm 1.5\end{array}$ & $\begin{array}{c}\text { LEED } \\
\text { Ion Bcattering } \\
\text { LEED } \\
\text { Ion scattering }\end{array}$ \\
\hline $\mathrm{Cu}(100)$ & $\begin{array}{l}\Delta d_{12} \\
\Delta d_{23}\end{array}$ & $\begin{array}{l}-3.7 \\
+1.9\end{array}$ & $\begin{array}{l}-1.4 \\
-0.3\end{array}$ & $\begin{array}{c}-2.1 \pm 1.7 \\
-1.1 \pm 0.4 \\
+0.45 \pm 1.7 \\
+1.7 \pm 0.6\end{array}$ & LEED \\
\hline $\mathrm{Cu}(111)$ & $\begin{array}{l}\Delta d_{12} \\
\Delta d_{2 s}\end{array}$ & $\begin{array}{l}-3.1 \\
+1.9\end{array}$ & $\begin{array}{l}-1.40 \\
-0.05\end{array}$ & $-0.7 \pm 0.5$ & LEED \\
\hline $\mathrm{Ag}(110)$ & $\begin{array}{l}\Delta d_{12} \\
\Delta d_{23}\end{array}$ & $\begin{array}{l}-6.0 \\
+2.8\end{array}$ & $\begin{array}{l}-5.7 \\
+0.3\end{array}$ & $\begin{array}{c}-5.7 \\
-7.8 \pm 2.5 \\
+2.2 \\
+4.3 \pm 2.5\end{array}$ & $\begin{array}{c}\text { LEED } \\
\text { Ion scattering } \\
\text { LEED } \\
\text { Ion scattering }\end{array}$ \\
\hline $\mathrm{Ag}_{\mathrm{g}}(\mathbf{1 0 0})$ & $\begin{array}{l}\Delta d_{12} \\
\Delta d_{2 s}\end{array}$ & $\begin{array}{l}-3.0 \\
+1.7\end{array}$ & $\begin{array}{l}-1.90 \\
-0.05\end{array}$ & & \\
\hline $\mathbf{A g}(111)$ & $\begin{array}{l}\Delta d_{12} \\
\Delta d_{23}\end{array}$ & $\begin{array}{l}-2.5 \\
+1.6\end{array}$ & $\begin{array}{l}-1.30 \\
-0.04\end{array}$ & & \\
\hline $\mathrm{Ni}(110)$ & $\begin{array}{l}\Delta d_{12} \\
\Delta d_{23}\end{array}$ & $\begin{array}{l}-7.6 \\
+3.4\end{array}$ & $\begin{array}{l}-4.87 \\
-0.57\end{array}$ & $\begin{array}{l}-8.7 \pm 0.5 \\
-9.0 \pm 1.0 \\
+3.0 \pm 0.6 \\
+3.5 \pm 1.5\end{array}$ & $\begin{array}{c}\text { LEED } \\
\text { Ion scattering } \\
\text { LEED } \\
\text { Ion scattering }\end{array}$ \\
\hline $\mathrm{Ni}(100)$ & $\begin{array}{l}\Delta d_{12} \\
\Delta d_{23}\end{array}$ & $\begin{array}{l}-3.7 \\
+2.0\end{array}$ & $\begin{array}{l}-0.002 \\
-.001\end{array}$ & $-3.2 \pm 0.5$ & Ion scattering \\
\hline $\mathrm{Ni}(111)$ & $\begin{array}{l}\Delta d_{12} \\
\Delta d_{23}\end{array}$ & $\begin{array}{l}-3.1 \\
+1.9\end{array}$ & $\begin{array}{l}-0.05 \\
+.00\end{array}$ & $-1.2 \pm 1.2$ & LEED \\
\hline $\operatorname{Al}(110)$ & $\begin{array}{l}\Delta d_{12} \\
\Delta d_{23}\end{array}$ & $\begin{array}{l}-10.4 \\
+4.7\end{array}$ & $\begin{array}{l}-10.4 \\
+3.1\end{array}$ & $\begin{array}{l}-8.6 \pm 0.8 \\
-8.5 \pm 1.0 \\
+5.0 \pm 1.1 \\
+5.5 \pm 1.1\end{array}$ & LEED \\
\hline $\operatorname{Al}(100)$ & $\begin{array}{l}\Delta d_{12} \\
\Delta d_{23}\end{array}$ & $\begin{array}{l}-4.9 \\
+1.8\end{array}$ & & & \\
\hline Al(111) & $\begin{array}{l}\Delta d_{12} \\
\Delta d_{23} \\
\end{array}$ & $\begin{array}{r}-3.9 \\
+2.5 \\
\end{array}$ & & $+0.9 \pm 0.7$ & LEED \\
\hline
\end{tabular}


TABLE V. - SURFACE ENERGIES IN ergs $/ \mathrm{cm}^{2}$ FOR Si(100)

[The $(2 \times 1)$ values are for symmetric dimer formation.]

\begin{tabular}{|c|c|c|c|c|c|}
\hline Surface & ECT & $\begin{array}{c}\text { Reference } \\
\text { a }\end{array}$ & $\begin{array}{c}\text { Reference } \\
\text { b }\end{array}$ & $\begin{array}{c}\text { Reference } \\
\text { c }\end{array}$ & $\begin{array}{c}\text { Reference } \\
\text { d }\end{array}$ \\
\hline Ideal & 2850 & 2740 & & & 2390 \\
$1 \times 1$ & 2820 & 2690 & & & 1970 \\
$2 \times 1$ & 1550 & & 1590 & 1610 & 1910 \\
\hline
\end{tabular}

a. M.T. Yin and M. Cohen, Phys. Rev. B 24, 2303 (1981).

b. K.C. Pandey in Proceedings of the seventh International Conference on Physics of Semiconductors, eds. D.F. Chadi and W.A. Harrison (Springer-Verlag, New York, 1985), p. 55.

c. N. Roberts and R.J. Needs, J. Phys. Cond. Mat. 1, 3139 (1989).

d. M.I. Baskes, J.S. Nelson, and A.F. Wright, Phys. Rev. B 40, 6085 (1989).

TABLE. VI - ENERGY BARRIER TO SLIP

$\mathrm{E}_{\mathrm{m}}(\mathrm{eV} /$ atom), MAXIMUM SLIP STRESS

$\sigma_{\mathrm{m}}\left(10^{10}\right.$ dynes $\left./ \mathrm{cm}^{2}\right)$ AND MEASURED

[a] MAXIMUM WHISKER STRENGTH

\begin{tabular}{|c|c|c|c|}
\hline \multicolumn{4}{|c|}{$\sigma_{\mathrm{s}}\left(10^{10}\right.$ dynes $\left./ \mathrm{cm}^{2}\right)$} \\
\hline $\mathrm{Ag}$ & 0.292 & 6.19 & 3.32 \\
$\mathrm{Ni}$ & .411 & 12.3 & 3.94 \\
$\mathrm{Cu}$ & .334 & 9.36 & 3.64 \\
$\mathrm{Al}$ & .225 & 4.34 & $\ldots$ \\
\hline
\end{tabular}

a. Whiskers, ed. J. Gordon Cook (Mills and Boon Limited, London, 1972) pp. 46-55. 


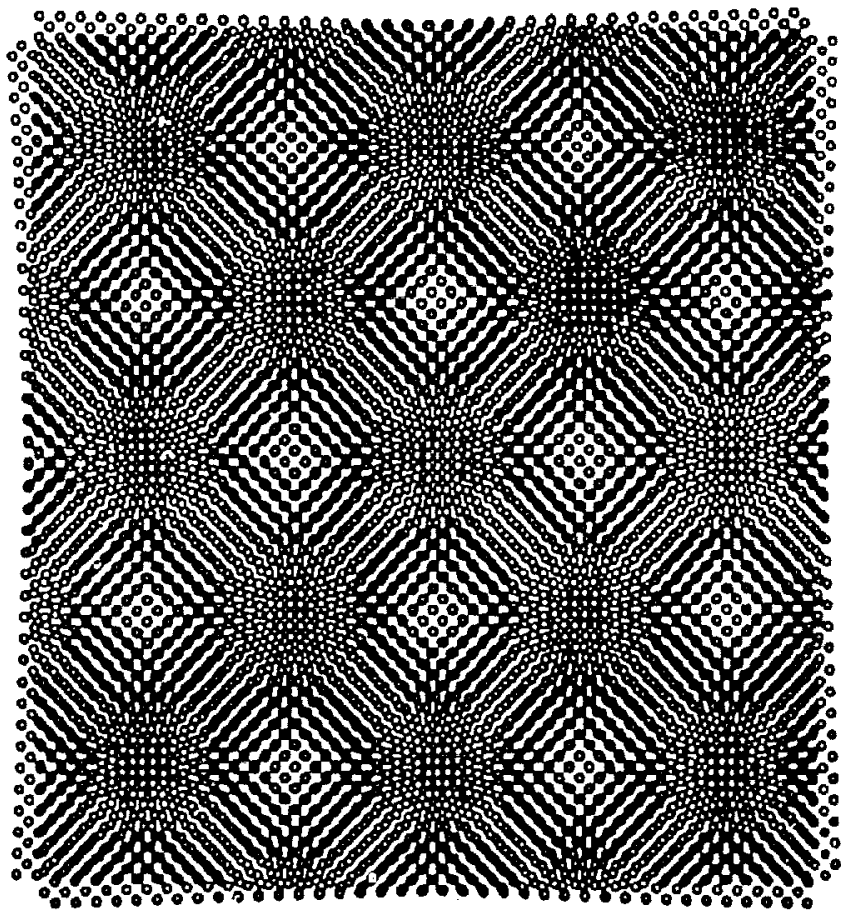

Flgure 1.-A $4^{\circ}$ rigid twist angle graln boundary for an fcc $(100)$ surface.

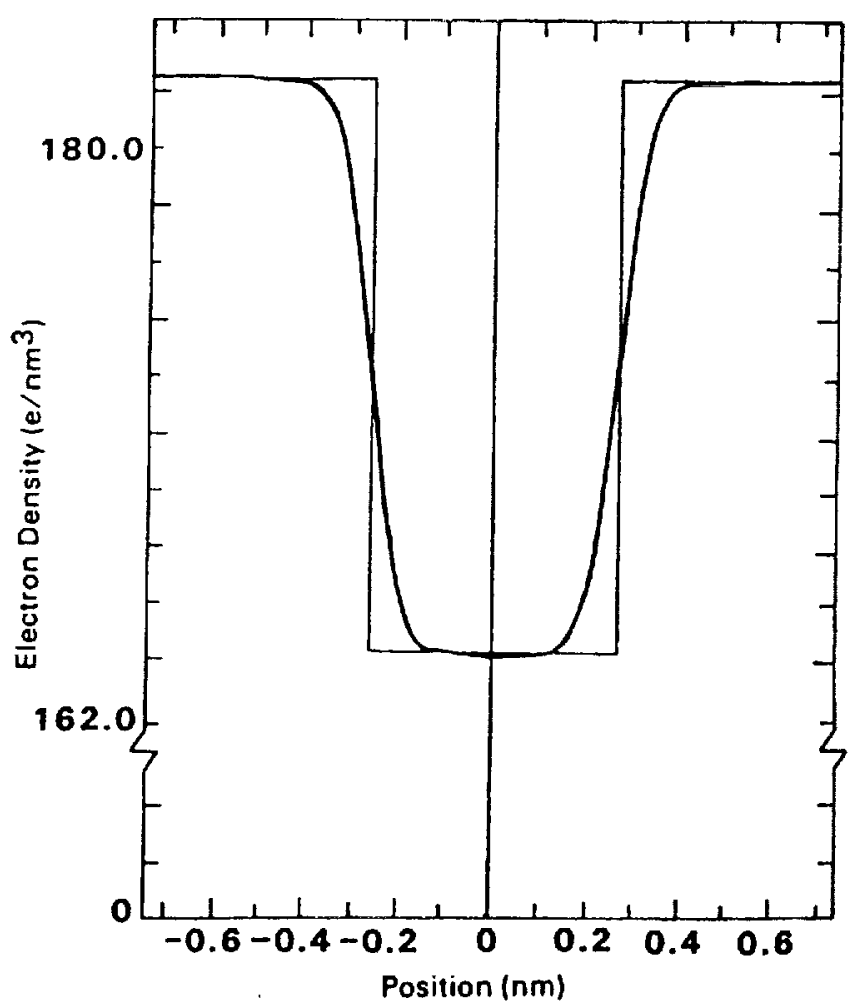

Figure 3.-A grain boundary represented by a dip in the jellium density where the square lines are tr:e jellium density and the Irregular the relaxed electron density.

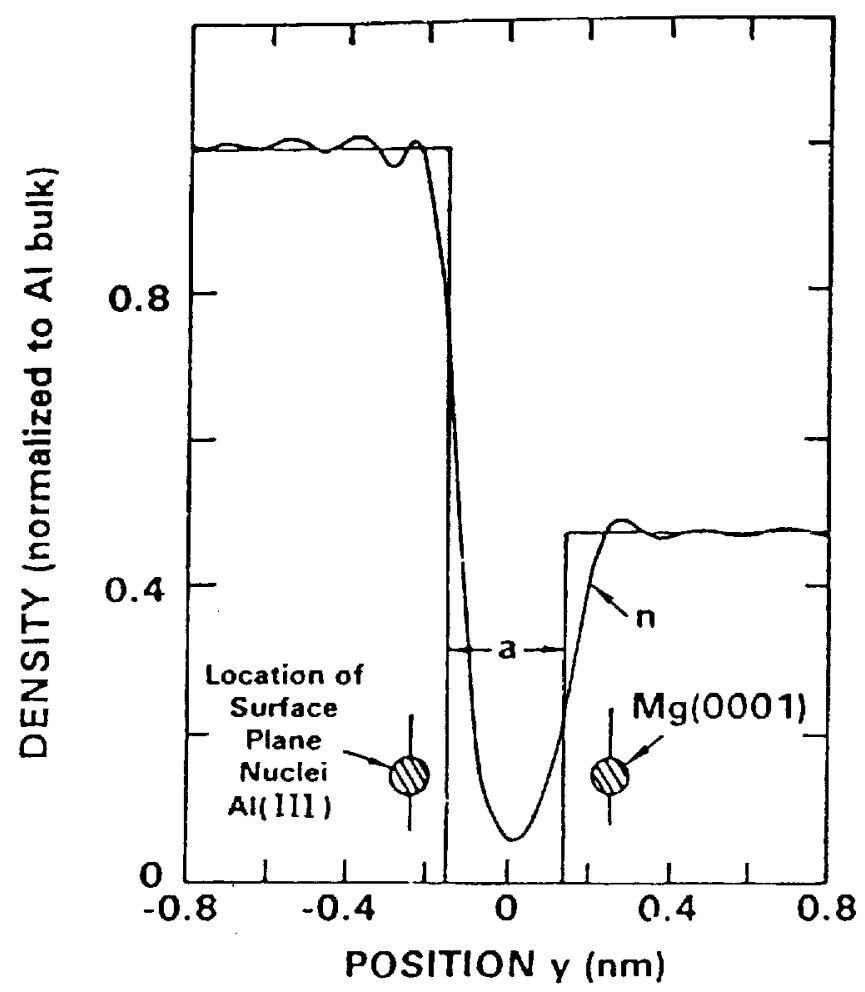

Figure 2.-A bimetallic Al-Mg interface where the square lines are the jellium density and the liregular lines the relaxed the electron density.

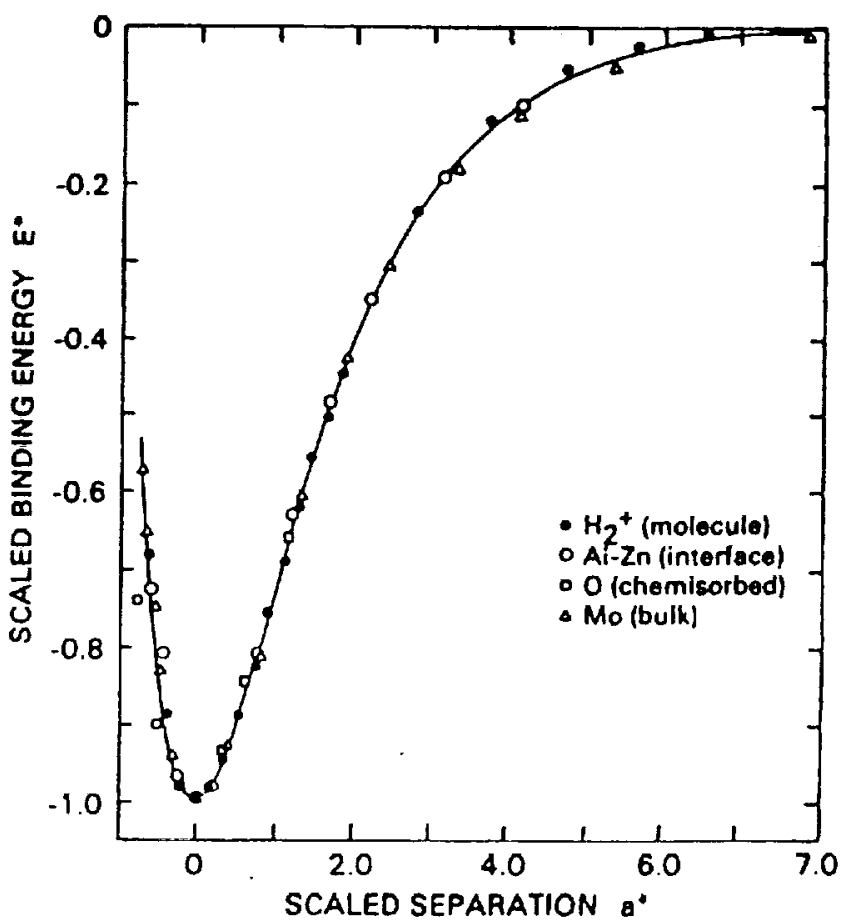

Figure 4.- Scaled binding energy as a function of scaled separation for the four systems shown. 


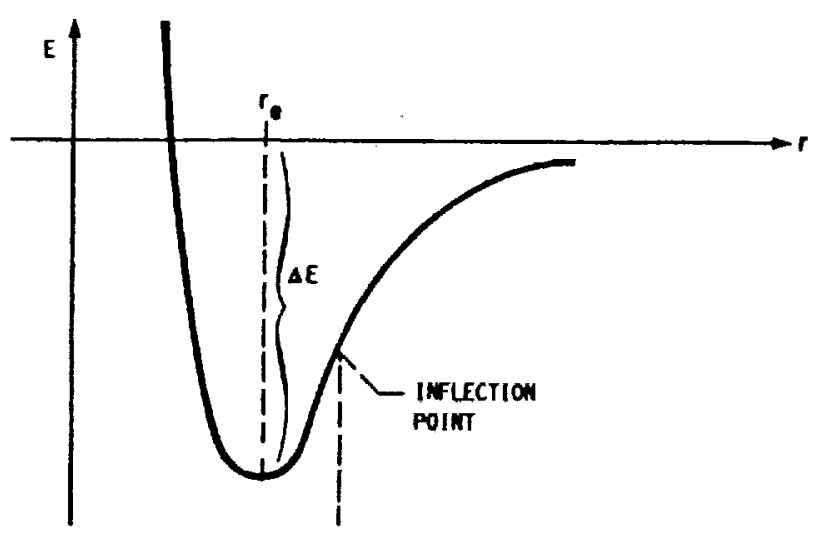

(a) Binding energy versus separation.

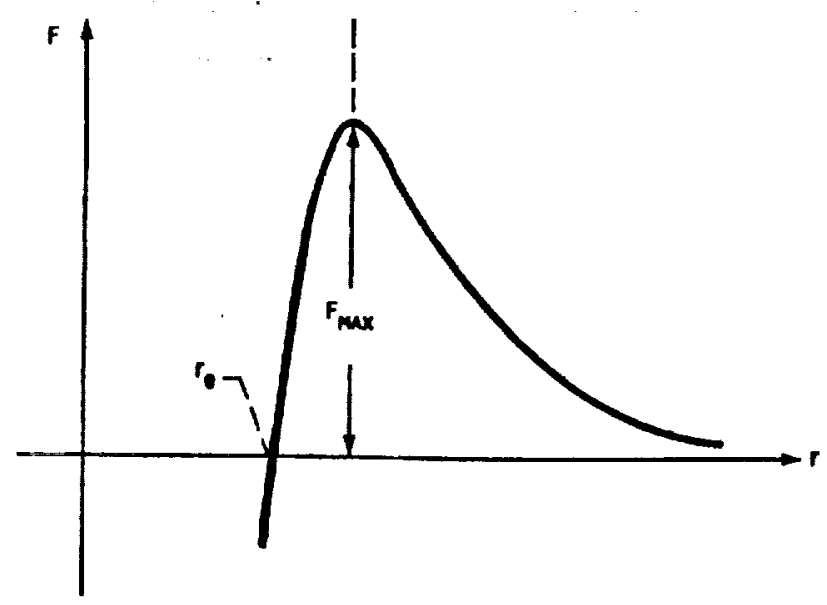

(b) Force versus separation.

Figure 5.-Example of a blnding energy and force curves.

ADHESIVE BINDING ENERGIES
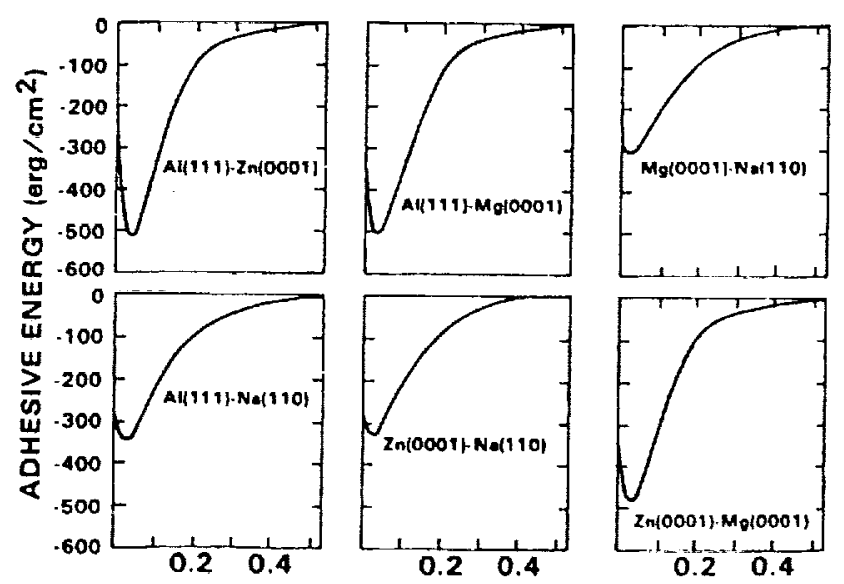

Flgure 6.-Adhesive binding energy versus the separation, $a$, between the surfaces indicated. 


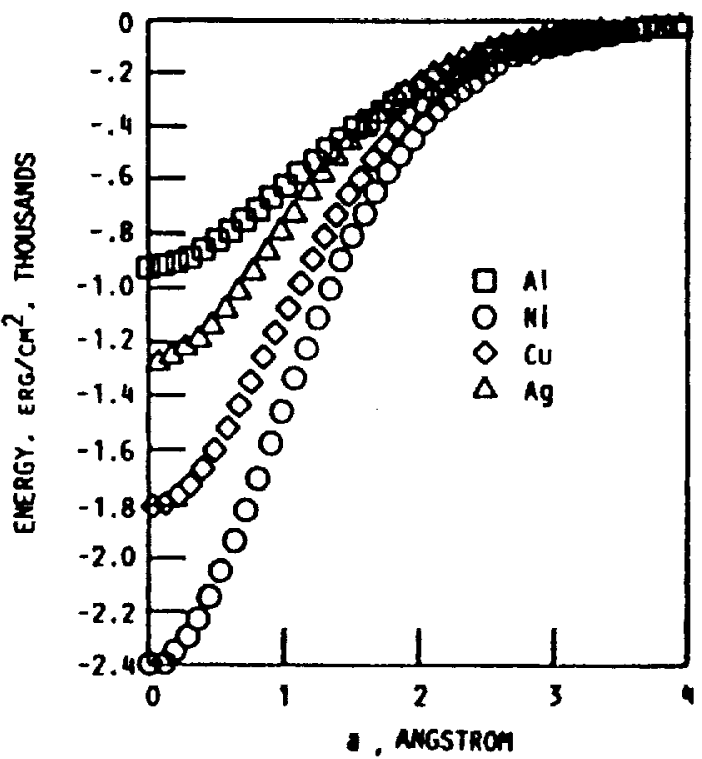

(a) For the (111) surface of $\mathrm{Al}, \mathrm{Ni}$, and $\mathrm{Ag}$.

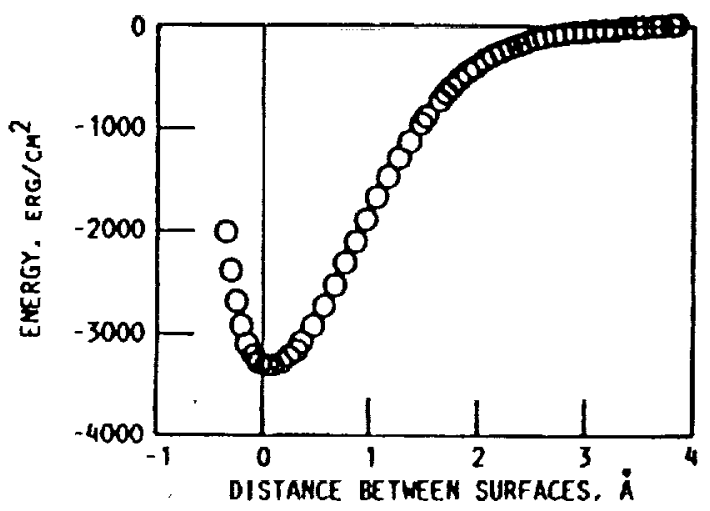

(b) For the $W(110)$ surface.

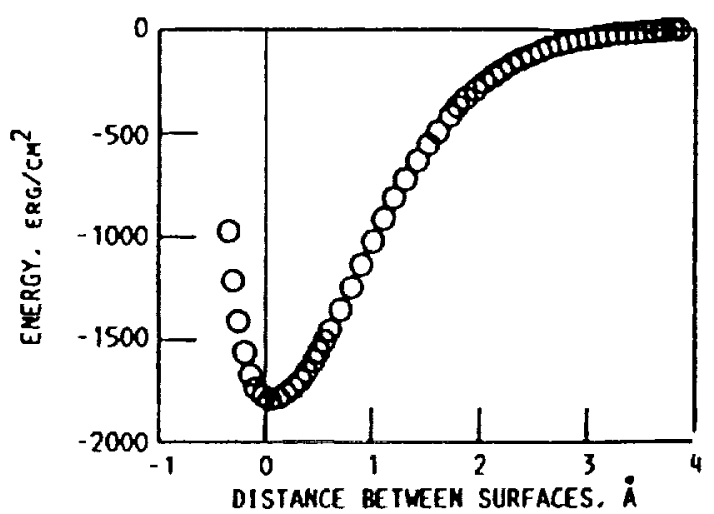

(c) For the $\mathrm{Fe}(110)$ surface.

Figure 8.-Rigid adhesive binding energy versus separation using ECT. 


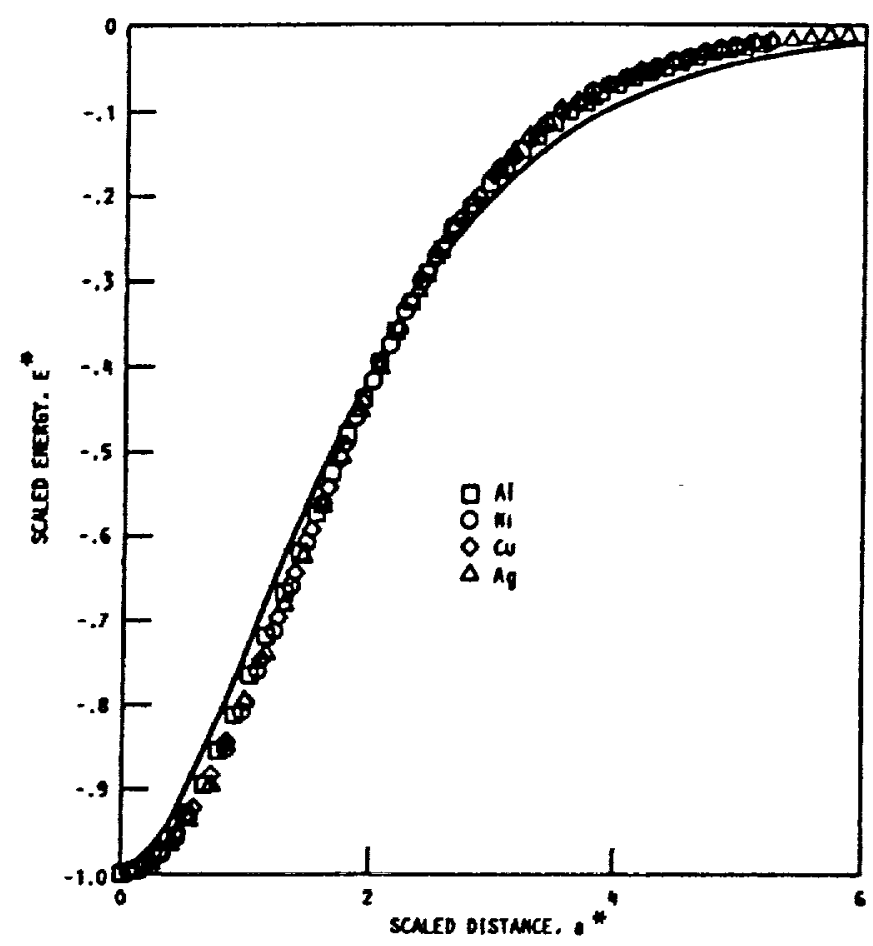

(a) fcc(111) Al, NI, Cu and Ag.

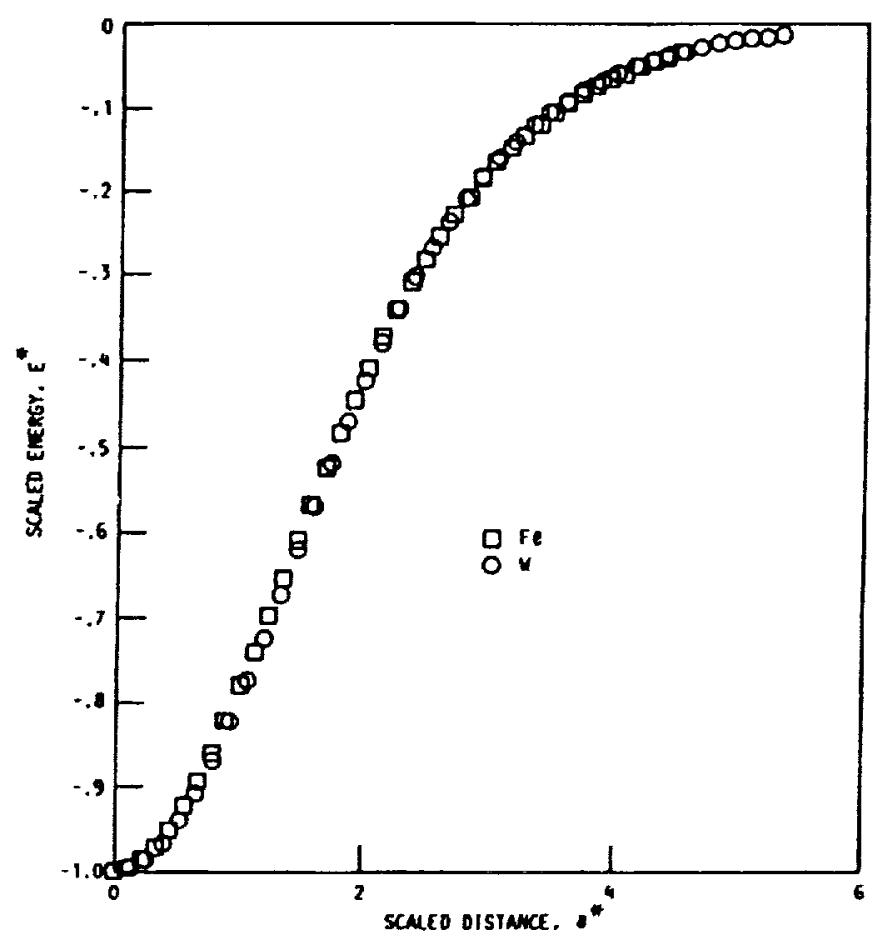

(b) bcc(110) W and Fe.

Figure 9.-Scaled adhesive energies from Flgure 8.

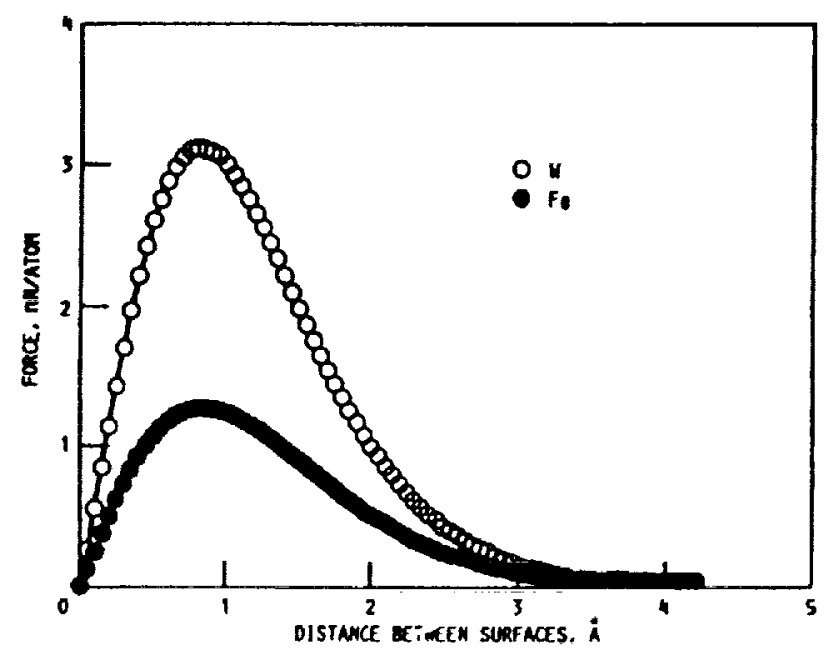

Figure 10.-Rigid adhesive blinding force versus separation for $W(110)$ and $F e(110)$ interfaces.

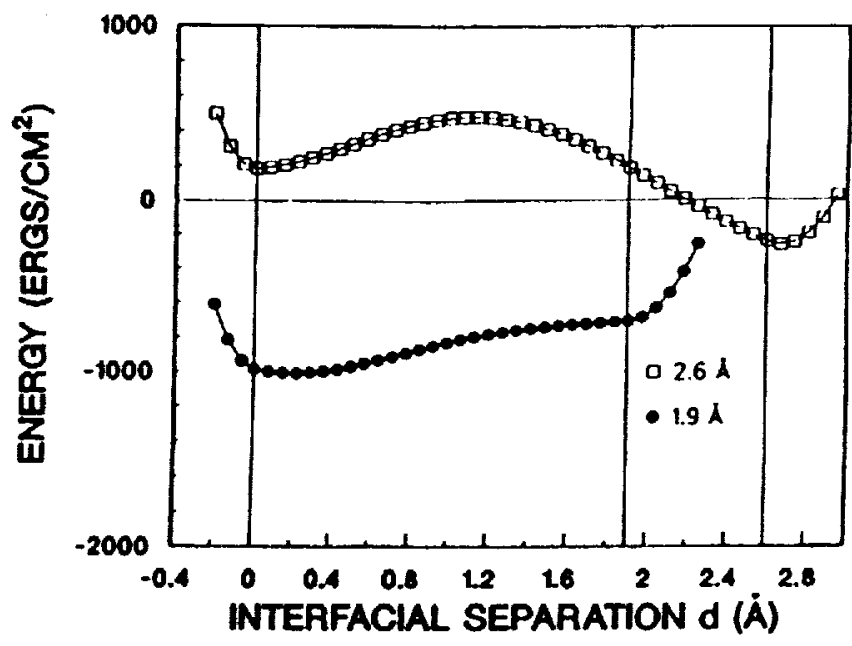

Figure 11. - Total energies as a functlon of symmetrlc movement of only the surface atomic layers for $\mathrm{Ni}(100)$ for two rigid separations. 


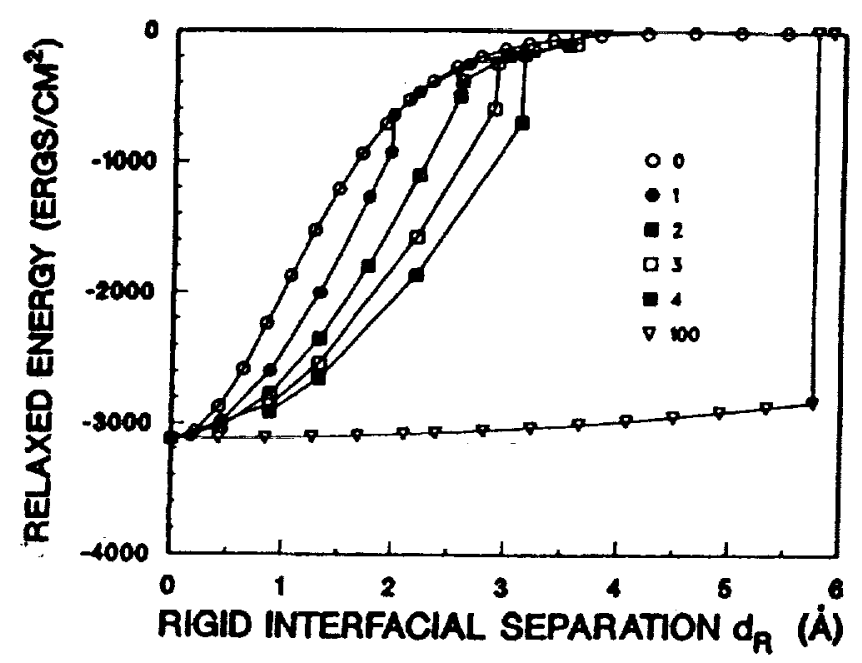

Flgure 12.-Relaxed and rigid adheslve binding energles for $N i(100)$ crystal surfaces in reglstry allowing different numbers of surface layers to move on each surface.

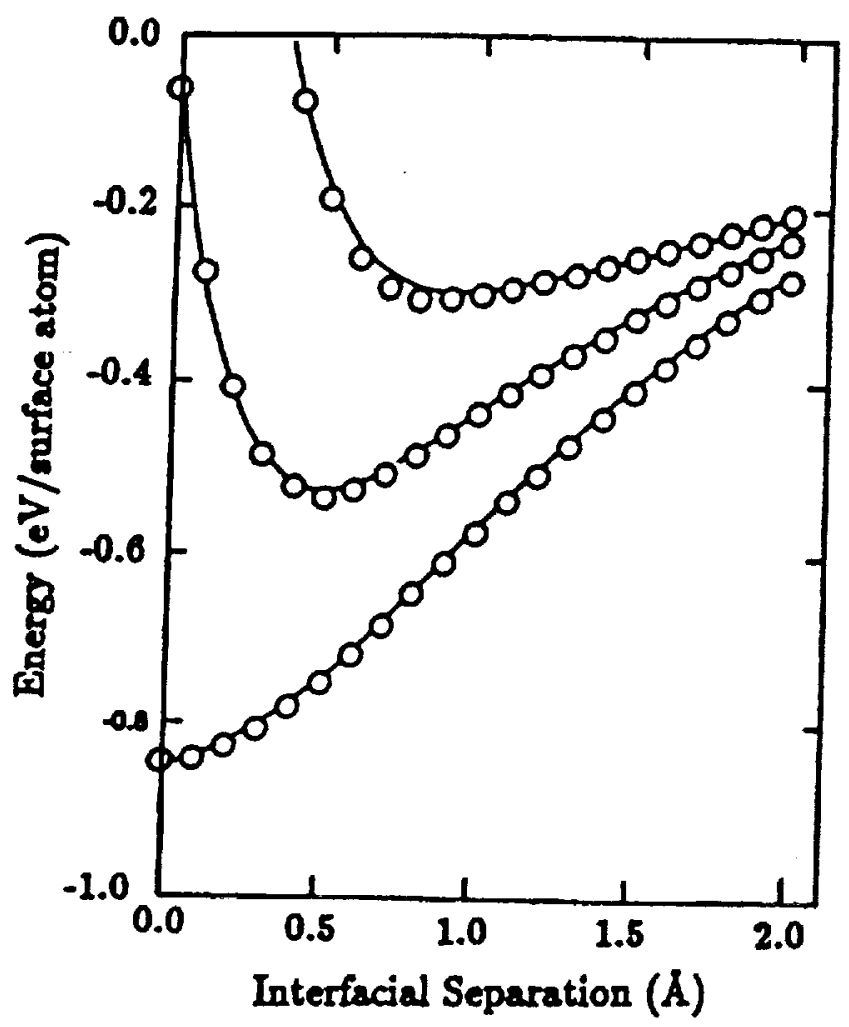

Figure 14.-Total cleavage energy as a function of interfacial separation for $A g(100)$ for different positions on the surface.

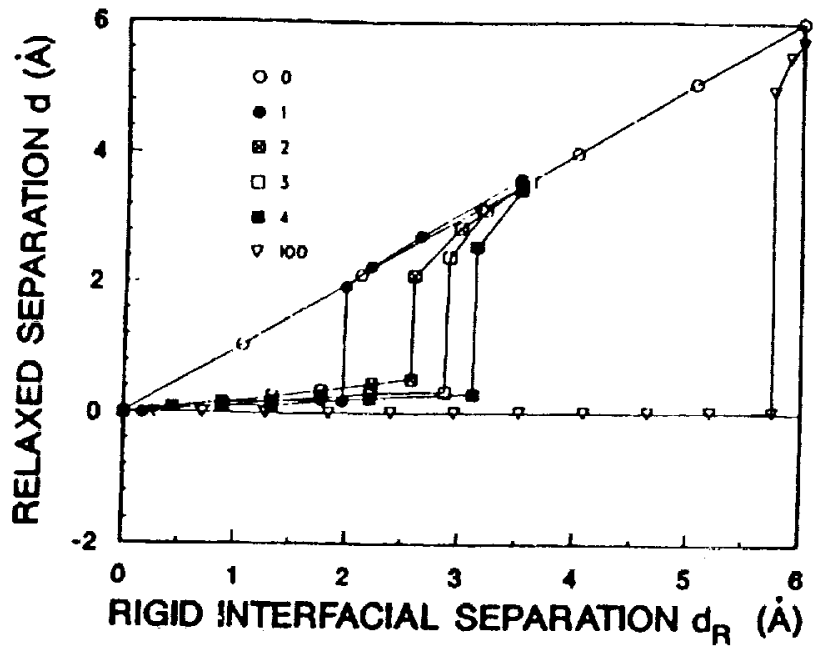

Figure 13.-Relaxed Interfacial separation $d$ as a function of rigid (unrelaxed) separation, $d_{h}$ for different number of layers allowed to relax.

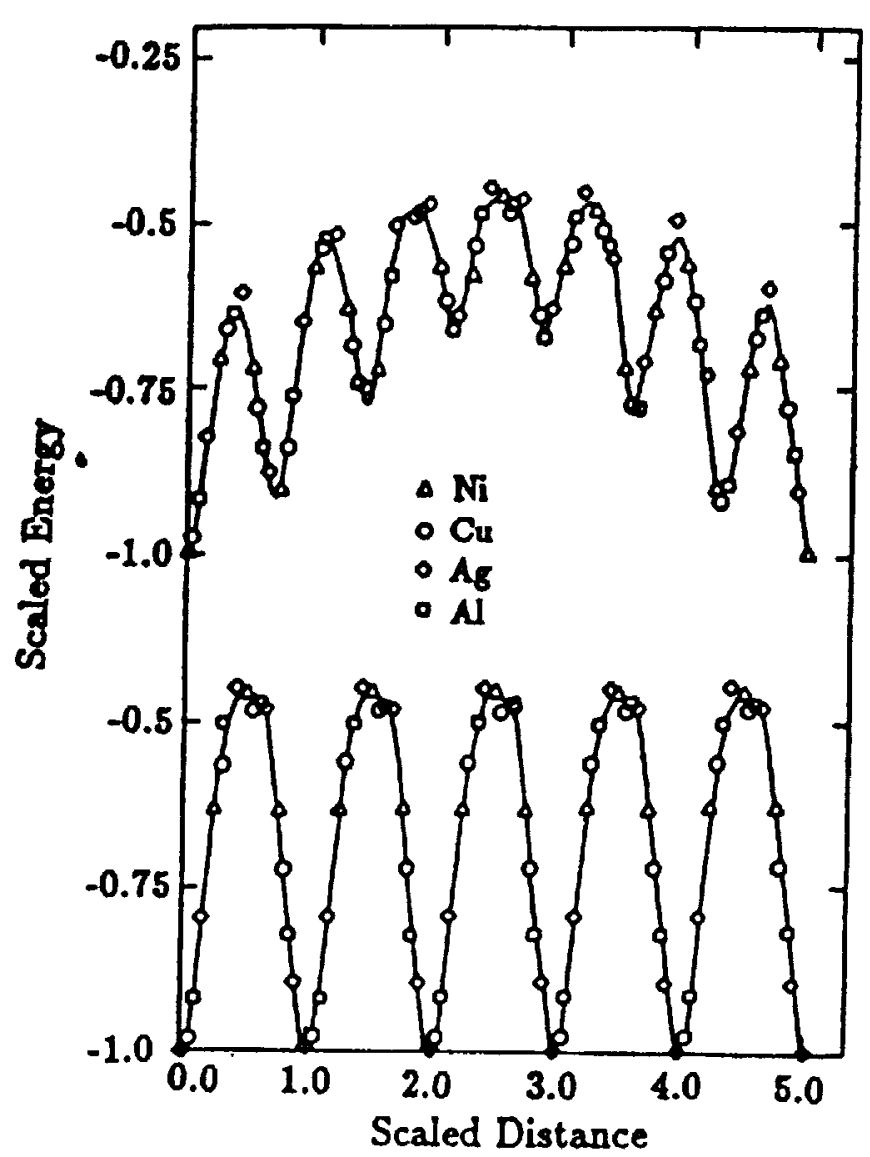

Figure 15.- Scaled total energy as a function of scaled slip distance for two different silp directions on (100) interface of $\mathrm{NI}, \mathrm{Cu}, \mathrm{Ag}$, and $\mathrm{Al}$. 


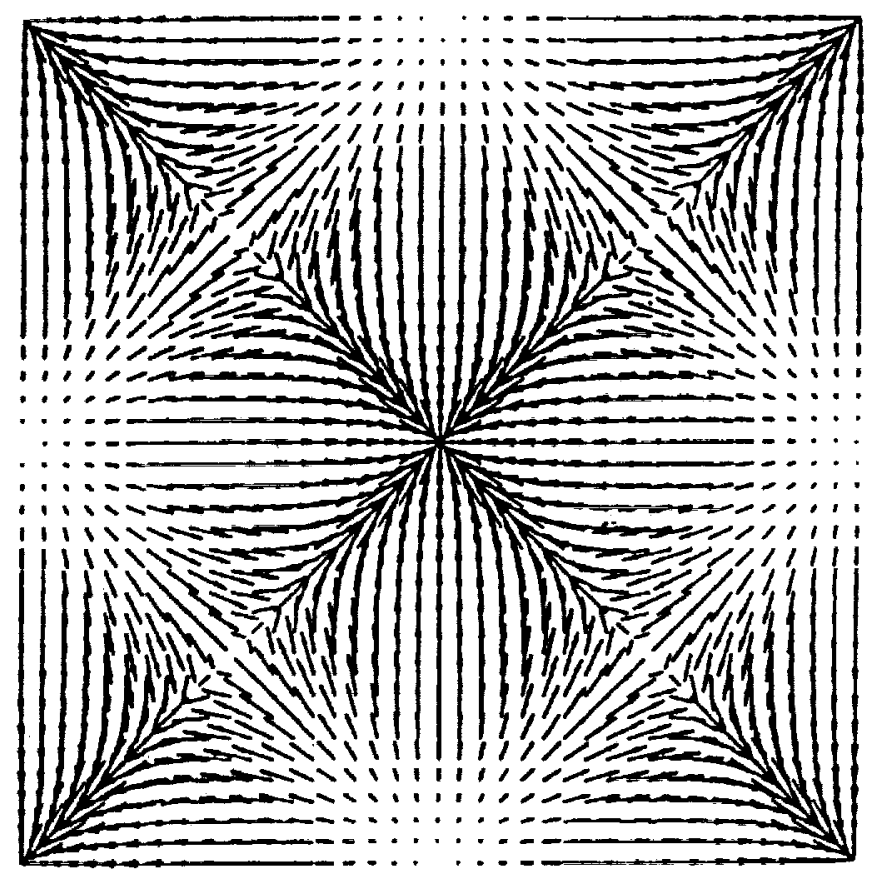

Figure 16. - A map of slip stresses for $A g$ crystals sliding on (100) surfaces at zero load.

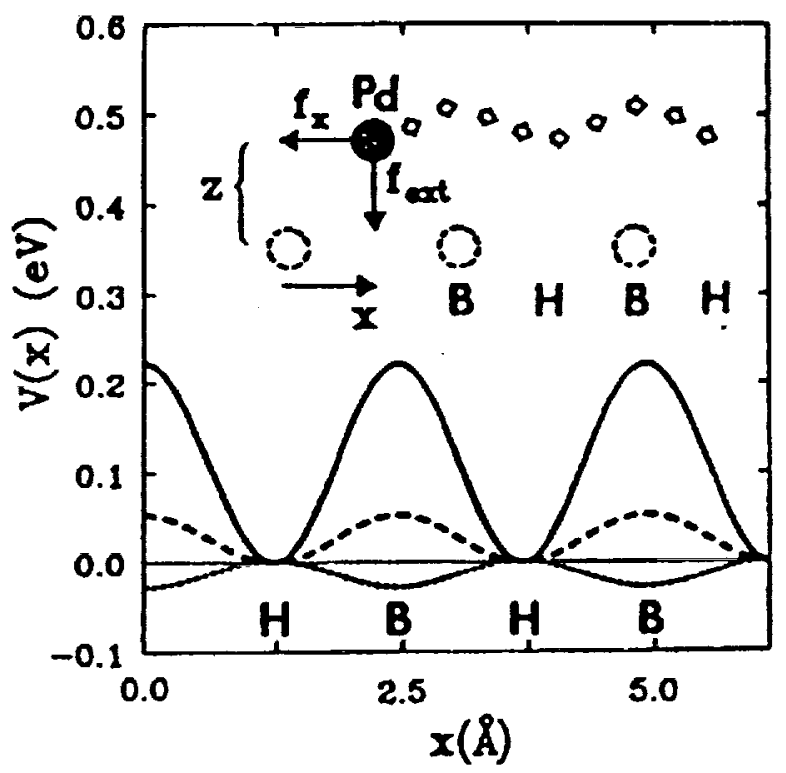

(a) Potential energy for a Pd-graphite Interface as a function of sliding position.

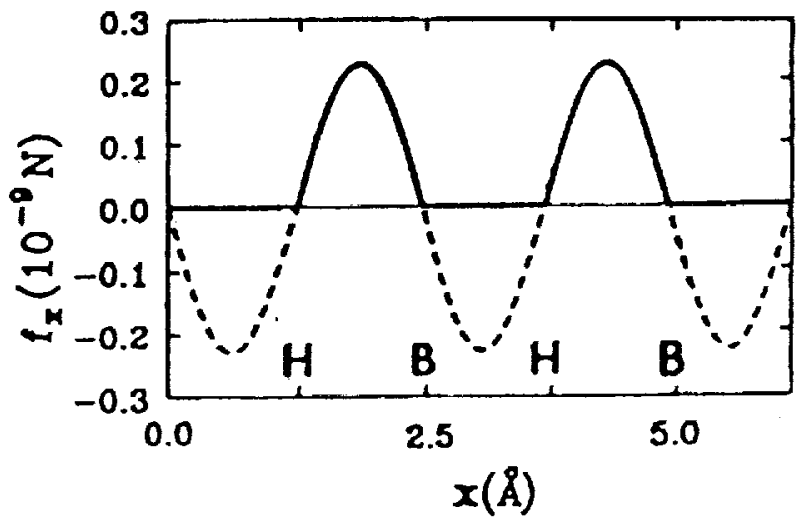

(b) Friction force as a function of position of the same interface [35].

Figure 17.-Interfacial potential energy and friction force. 


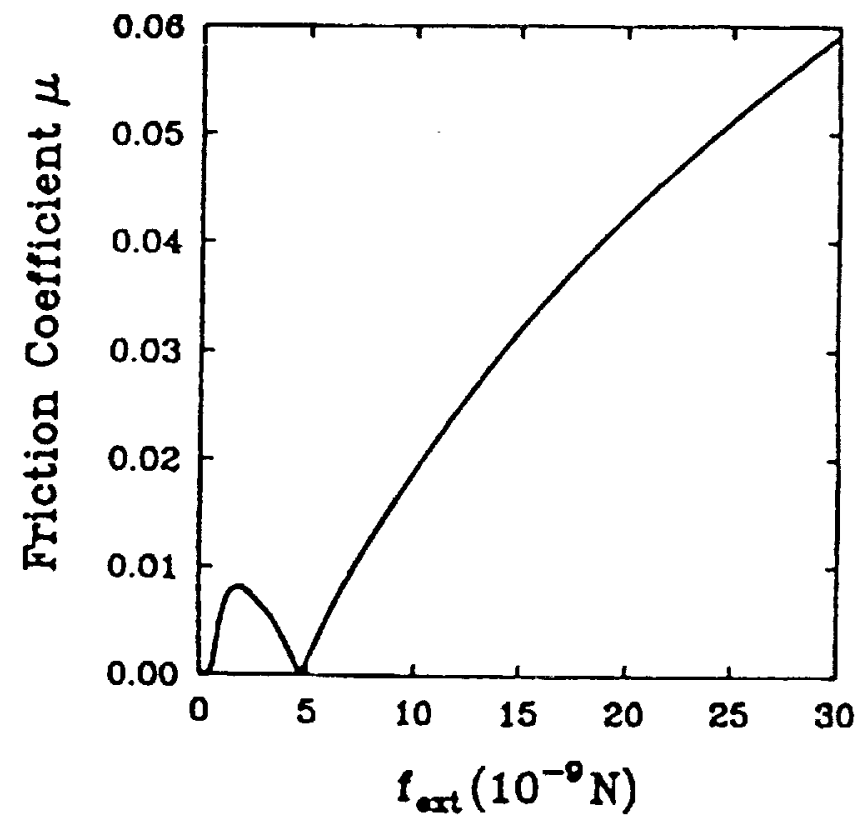

Figure 18.-Microscoplc friction coefficient $\mu$ as a function of the external force per atom $f_{\text {ext }}$.

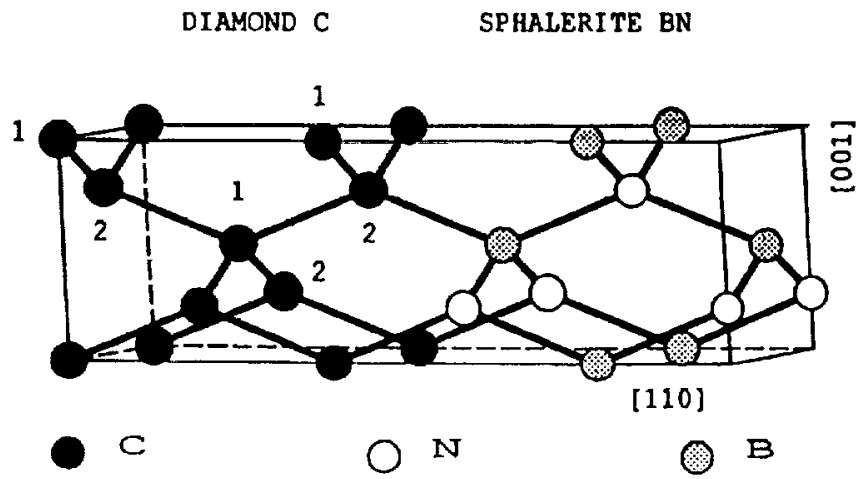

Figure 19. - Crystal structure of the $\mathrm{C} 2(110)$ and $\mathrm{BN}(001) 1 \times 1$ superlattice [39]. 


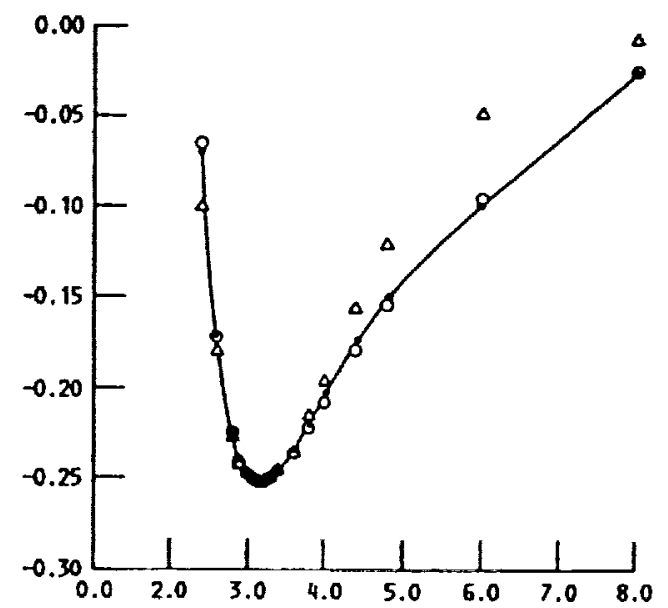

(a) A1F.

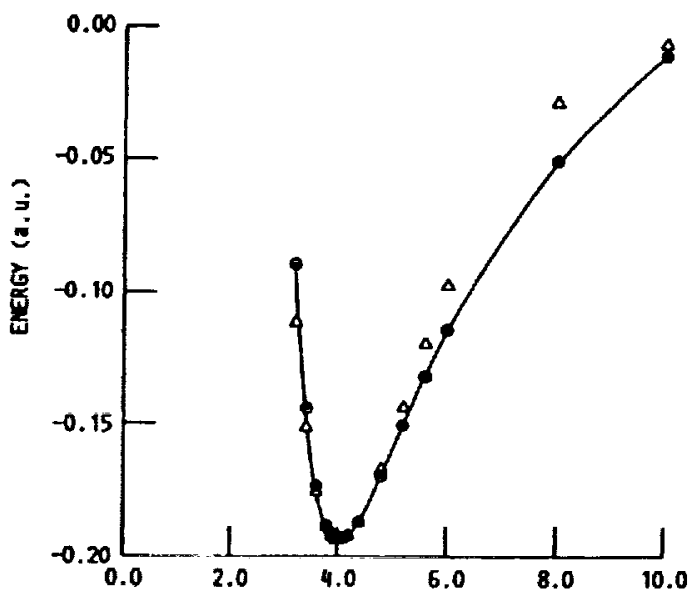

(b) A1C1.

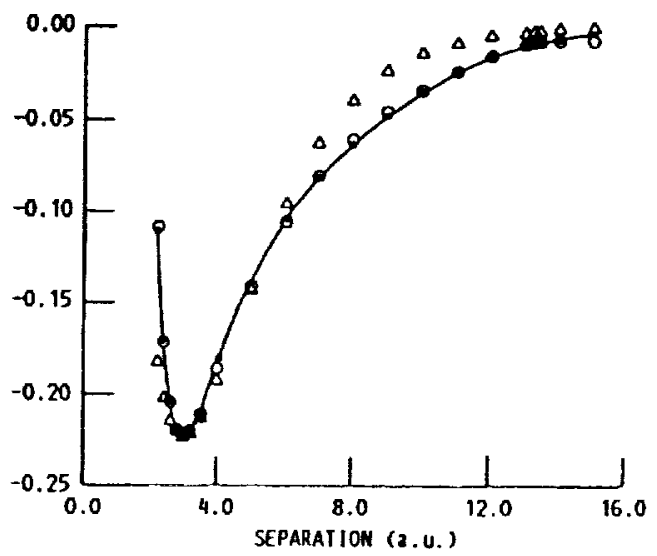

(c) LiF.

Figure 20.-A comparison between first-principles potential curves $(O)$, the Rydberg function ( $\Delta$ ) and eq. 51( $\longrightarrow$ ). 


\begin{tabular}{|c|c|c|c|}
\hline \multicolumn{3}{|c|}{ REPORT DOCUMENTATION PAGE } & $\begin{array}{l}\text { Form Approved } \\
\text { OMB No. 0704-0188 }\end{array}$ \\
\hline \multicolumn{4}{|c|}{ 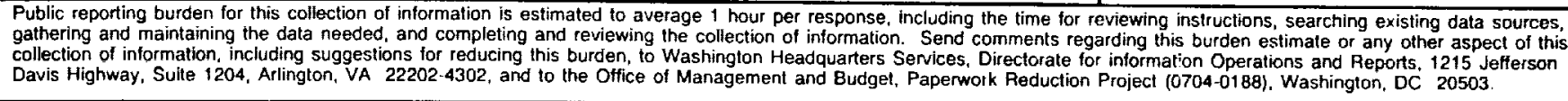 } \\
\hline 1. AGENCY USE ONLY (Leave blank) & $\begin{array}{r}\text { 2. REPORT DATE } \\
1992\end{array}$ & \multicolumn{2}{|c|}{$\begin{array}{r}\text { 3. REPORT TYPE AND DATES COVERED } \\
\text { Technical Memorandum } \\
\end{array}$} \\
\hline \multicolumn{3}{|c|}{$\begin{array}{l}\text { 4. TITLE AND SUBTITLE } \\
\text { Computational Techniques in Tribology and Material Science at the } \\
\text { Atomic Level }\end{array}$} & \multirow{2}{*}{$\begin{array}{l}\text { 5. FUNDING NUMBERS } \\
\text { WU }-505-90-53\end{array}$} \\
\hline \multicolumn{3}{|c|}{$\begin{array}{l}\text { 6. AUTHOR(S) } \\
\text { J. Ferrante and G.H. Bozzolo }\end{array}$} & \\
\hline \multicolumn{3}{|c|}{$\begin{array}{l}\text { 7. PERFORMING ORGANIZATION NAME(S) AND ADDRESS(ES) } \\
\text { National Acronautics and Space Administration } \\
\text { Lewis Research Center } \\
\text { Cleveland, Ohio } 44135-3191\end{array}$} & $\begin{array}{l}\text { 8. PERFORMING ORGANIZATION } \\
\text { REPORT NUMBER }\end{array}$ \\
\hline \multicolumn{3}{|c|}{$\begin{array}{l}\text { 9. SPONSORING/MONITORING AGENCY NAMES(S) AND ADDRESS(ES) } \\
\text { National Aeronautics and Space Administration } \\
\text { Washington, D.C. } 20546-0001\end{array}$} & $\begin{array}{l}\text { ONSORING/MONITORING } \\
\text { ENCY REPORT NUMBER } \\
\text { SSA TM- } 105573\end{array}$ \\
\hline \multicolumn{4}{|c|}{$\begin{array}{l}\text { Prepared for the NATO Research Institute Workshop on Fundamentals of Friction sponsored by the North Atlantic Treaty } \\
\text { Organization, Braunlage, Germany, August 5-19, 1991. J. Ferrante, NASA Lewis Research Center; G.H. Bozzolo, Analex } \\
\text { Corporation, } 3001 \text { Aerospace Parkway, Brook Park, Ohio 44142. Responsible person, J. Ferrante, (216) 433-6069. }\end{array}$} \\
\hline \multicolumn{3}{|c|}{$\begin{array}{l}\text { 12a. DISTRIBUTION/AVAILABILITY STATEMENT } \\
\text { Unclassified-Unlimited } \\
\text { Subject Category } 70\end{array}$} & 12b. DISTRIBUTION CODE \\
\hline \multicolumn{4}{|c|}{$\begin{array}{l}\text { 13. ABSTRACT (Maximum } 200 \text { words) } \\
\text { Computations in tribology and material science at the atomic level present considerable difficulties. In this paper } \\
\text { computational techniques ranging from first-principles to semi-empirical and their limitations are discussed. Example } \\
\text { calculations of metallic surface energies using semi-empirical techniques are presented. Finally, application of the } \\
\text { methods to calculation of adhesion and friction are presented. }\end{array}$} \\
\hline \multirow{2}{*}{\multicolumn{2}{|c|}{$\begin{array}{l}\text { 14. SUBJECT TERMS } \\
\text { Friction; Adhesion; Atomic-potentials }\end{array}$}} & & $\begin{array}{l}\text { 15. NUMBER OF PAGES } \\
30\end{array}$ \\
\hline & & & $\begin{array}{r}\text { 16. PRICE CODE } \\
\text { A03 }\end{array}$ \\
\hline $\begin{array}{l}\text { 17. SECURITY CLASSIFICATION } \\
\text { OF REPORT } \\
\text { Unclassified }\end{array}$ & $\begin{array}{l}\text { 18. SECURITY CLASSIFICATION } \\
\text { OF THIS PAGE } \\
\text { Unclassified }\end{array}$ & $\begin{array}{l}\text { 19. SECURITY CLASSIFICATION } \\
\text { OF ABSTHACT } \\
\text { Unclassified }\end{array}$ & 20. LIMITATION OF ABSTRACT \\
\hline NSN 7540-01-280-5500 & & & $\begin{array}{l}\text { ndard Form } 298 \text { (Rev. 2-89) } \\
\text { scribed by ANSI Std. Z39-18 } \\
-102\end{array}$ \\
\hline
\end{tabular}

